\title{
El impacto de la deforestación en la erosión de la cuenca del río Magdalena (1980-2010)
}

\author{
Juan D. Restrepo A. \\ Departamento de Ciencias de la Tierra, Escuela de Ciencias, Universidad EAFIT, Medellín, Colombia
}

\begin{abstract}
Resumen
En la última década, la cuenca del río Magdalena ha experimentado un incremento en las tasas de erosión del orden del $34 \%$, pasando de $550 \mathrm{t} \mathrm{km}^{-2} \mathrm{a}^{-1}$ antes del año 2000 a $710 \mathrm{t} \mathrm{km}^{-2} \mathrm{a}^{-1}$ en el periodo 2000-2010, con un aumento en el transporte total de sedimentos de $44 \mathrm{Mt} \mathrm{a}^{-1}$. El análisis de la variabilidad espacial de la producción de sedimentos indica que el $78 \%$ de la cuenca se encuentra en estado crítico de erosión dada la pérdida de bosques primarios de más del 60 \% en el periodo entre 1980 y 2010. Mediante la calibración del modelo BQART para el periodo de 1980 a 2010, que combina variables climáticas, hidrológicas, litológicas, morfométricas y del impacto humano por deforestación, se explicó el 86 \% de la variabilidad del transporte de sedimentos en la cuenca. El 9 \% del transporte de sedimentos acumulado de las tres últimas décadas se debió a la deforestación; cerca de 160 Mt de sedimentos se han generados por la deforestación entre el 2000 y el 2010. Los indicadores económicos del Magdalena en las seis últimas décadas señalan que las actividades agrícolas fueron la principal causa del cambio en el uso de los suelos y de la pérdida forestal. El deterioro de los suelos de la cuenca requiere de medidas a escala regional, y no solamente de planes de mitigación ante el cambio climático.
\end{abstract}

Palabras clave: río Magdalena, deforestación, transporte de sedimentos, erosión.

The impact of deforestation on the erosion in the Magdalena River drainage basin (1980-2010)

\begin{abstract}
During the last decade, the Magdalena River drainage basin has witnessed an increase in erosion rates of 34\%, from $550 \mathrm{t} \mathrm{km}^{-2} \mathrm{y}^{-1}$ before 2000 to $710 \mathrm{t} \mathrm{km}^{-2} \mathrm{y}^{-1}$ for the 2000-2010 yr-period, and the average sediment load for the whole basin increased in $44 \mathrm{Mt} \mathrm{y}^{-1}$ for the same period. Sediment yield spatial analysis indicated that $78 \%$ of the catchment is under strong erosional conditions due to the clearance of more than $60 \%$ of the natural forest between 1980 and 2010. Sediment load simulation by using the BQART, a robust model that combines climatic, morphometric, hydrological, lithologic and human induced variables, and explains $86 \%$ of the sediment load variability, indicated that $9 \%$ of the combined sediment load during the last three decades was due to deforestation; about $160 \mathrm{Mt}$ have been produced by forest clearance between 2000 and 2010. The trends in economic indicators show that agriculture was the main cause of land-use change and, consequently, of forest loss within the basin. Soil deterioration and increasing trends in erosion require capacity building in environmental governance in addition to climate change mitigation policies.
\end{abstract}

Key words: Magdalena River, deforestation, sediment load, erosion.

\section{Introducción}

En los últimos años se ha constatado un apreciable aumento en la frecuencia y magnitud de los procesos peligrosos relacionados con los efectos de las lluvias en la cuenca del río Magdalena, y especialmente en los daños humanos y materiales, directos e indirectos, que tales procesos producen. Lo ocurrido en el invierno de 2010 a 2011 fue un ejemplo particularmente revelador de lo que parece ser una tendencia de fondo, que constituye una fuente importante de adversidades para la población, con consecuencias muy negativas para la economía y el desarrollo del país.
Aunque con frecuencia el aumento en este tipo de desastres se atribuye al cambio climático, existen serias evidencias, tanto en Colombia (Restrepo \& Syvitski, 2006; Restrepo, 2008) como a nivel global ( Rivas, et al., 2006; Bonachea, et al., 2010; Syvitski \& Kettner, 2011), de que la causa principal son las alteraciones producidas sobre la superficie terrestre por la deforestación, la agricultura, la minería, la

\footnotetext{
Correspondencia:

Juan D. Restrepo A., jdrestre@eafit.edu.co

Recibido: 29 de octubre de 2014

Aceptado: 26 de marzo de 2015
} 
expansión urbana y la construcción de infraestructura. Visto de otra forma, la aceleración de los procesos hidrogeológicos superficiales en las cuencas hidrográficas como la denudación de los suelos, la generación de sedimentos, los deslizamientos, las inundaciones y la colmatación de ríos y de masas de agua, pueden ser producto del cambio climático o de la intervención humana, o de ambos factores. Este incremento en los procesos geológicos que afectan la superficie terrestre y representan alto riesgo para las comunidades es evidente y se ha comprobado a escala global. La comunidad científica mundial todavía trabaja para determinar si estamos ante un "cambio geomorfológico global” independiente del cambio climático y que se sumaría a este (Cendrero, et al., 2006; Bonachea, et al., 2010).

En el marco de estos escenarios de intervención humana sobre los suelos, es de esperarse que haya una importante reducción de la resiliencia de los sistemas naturales ante distintos agentes desestabilizadores (lluvias intensas y acciones humanas), así como una intensificación de los procesos geológicos superficiales causantes de los desastres. De confirmarse lo anterior, habría consecuencias importantes en la formulación de estrategias de mitigación de los desastres, ya que el foco de las medidas no debería orientarse exclusivamente hacia el cambio climático, sino hacia el control en la degradación de los suelos. El primero depende sobre todo de políticas internacionales, mientras que el segundo es mucho más fácil de abordar y gestionar a nivel nacional (Cendrero, et al., 2004).

En la cuenca del río Magdalena (Figura 1), así como en otros sistemas hidrográficos a nivel mundial, las consecuencias de la erosión de los suelos incluyen efectos directos como la generación de sedimento, la reducción de la capacidad productiva del suelo, los deslizamientos, la colmatación de ríos y masas de agua y las inundaciones, con consecuencias lógicas sobre la seguridad y la salud de la población y sobre el sistema productivo. En este escenario de impacto ambiental es importante determinar en qué medida el conjunto de acciones que degradan el territorio (incluido el suelo en el sentido edafológico y las interacciones entre agua y superficie terrestre), están en la raíz de los impactos mencionados. El análisis permitirá, por lo tanto, orientar mejor las políticas y estrategias de mitigación de desastres.

Este artículo está encaminado a clarificar dichos aspectos en términos del impacto humano en la erosión del río Magdalena, principalmente para establecer qué porcentaje de la erosión en la cuenca ha sido producto de la deforestación en las últimas tres décadas (1980-2010). El análisis se basa en: 1) estudios anteriores sobre las tendencias de la erosión en la cuenca del río Magdalena para el periodo 1980-2000
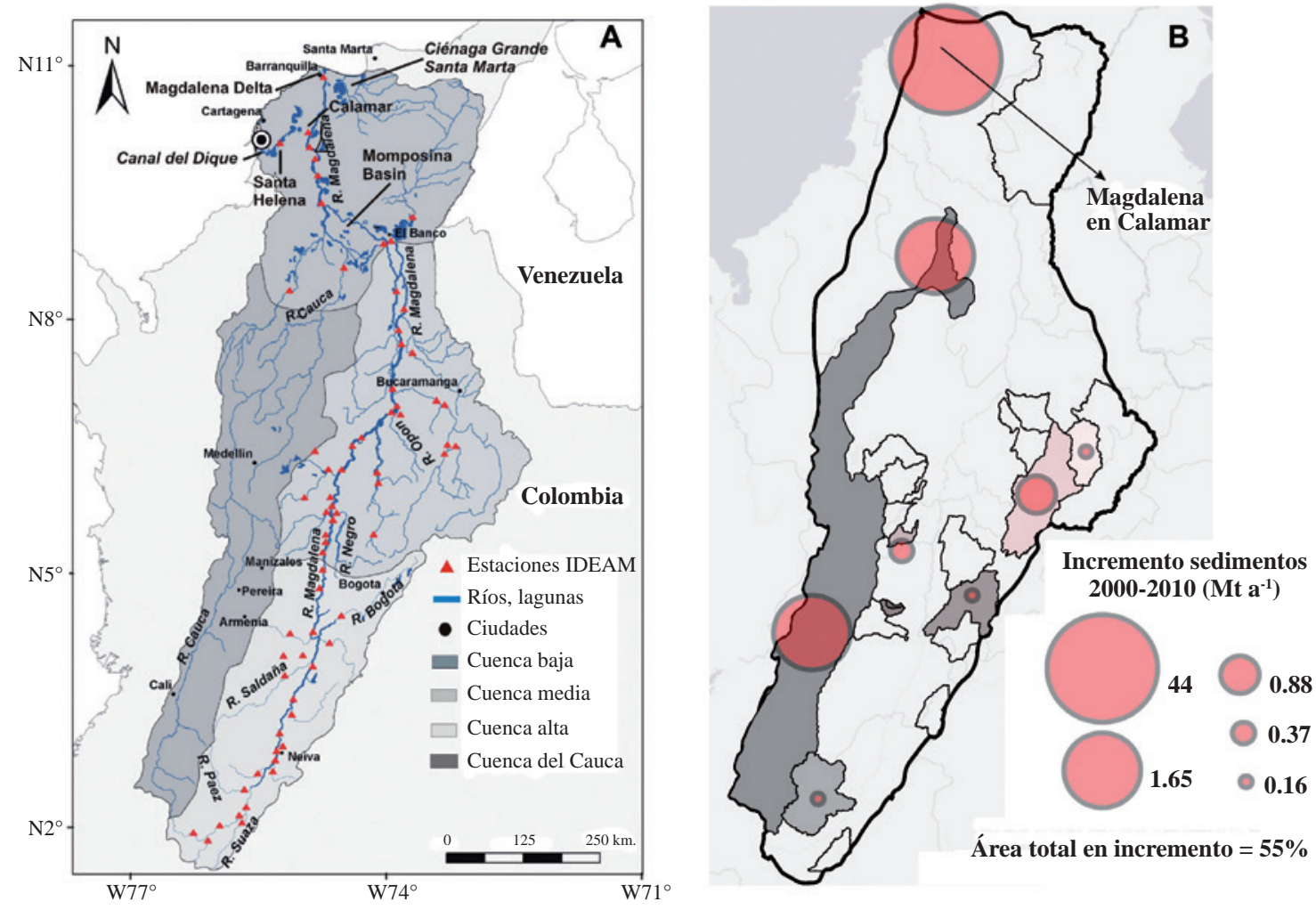

Figura 1. Mapa de la cuenca del río Magdalena que muestra las estaciones de aforo de transporte de sedimentos analizadas en estudios anteriores (Restrepo, 2005; Restrepo \& Syvitski, 2006; Restrepo, et al., 2006a, 2006b; Restrepo, 2008; Kettner, et al., 2010) (A) y mapa de cuencas con incrementos en el transporte de sedimentos durante el periodo 2000-2010. 
(Restrepo, 2005; Restrepo \& Restrepo, 2005; Restrepo \& Syvitski, 2006; Restrepo, et al., 2006a, 2006b; Restrepo, 2008) (Figura 1); 2) en la simulación numérica del transporte de sedimentos mediante la aplicación del modelo BQART (Kettner, et al., 2010), y 3) en resultados recientes de la aplicación del modelo BQART con datos sobre el transporte de sedimentos y la deforestación hasta el 2010.

Desde la pasada emergencia invernal (2010-2011) el gobierno ha estado identificando las líneas de investigación fundamentales que sirvan de base para hacer más eficientes las inversiones de capital en la mitigación de inundaciones o en la infraestructura fluvial, incluida la construcción de diques, puentes y defensas hidráulicas. Si algo es cierto es que el país pide inversión ya mismo, pero para que esta no se malgaste se requieren proyectos de investigación ambiental que permitan poner en práctica soluciones de largo plazo, abordando las causas de las inundaciones de los ríos colombianos y no simplemente los síntomas: "Después de la atención de la emergencia, el primer paso para una solución sostenible sería un adecuado entendimiento de lo que ha sucedido. Un poco de historia podría servir para no repetirla." (Editorial de El Espectador, 1 de mayo de 2011).

La estimación de los valores históricos y de los futuros escenarios de erosión en la cuenca del río Magdalena como producto de la deforestación, son una de las herramientas principales en la orientación de políticas nacionales para el control de la degradación de los suelos y de la mitigación de desastres naturales asociados a la erosión (deslizamientos e inundaciones). De igual manera, los valores de la producción y el transporte de sedimentos en el sistema del río Magdalena debidos a la deforestación, permitirán hacer futuras evaluaciones económicas de los costos ambientales de la erosión en Colombia.

De acuerdo con el informe ambiental para la reducción de la pobreza del Banco Mundial (Sánchez-Triana, et al., 2007), Colombia no cuenta con las políticas ni el marco institucional para la protección de los suelos. Parte de dicha carencia de normas y planes de mitigación contra la erosión radica en la falta de conocimiento sobre cómo hemos alterado los suelos y cuáles han sido las causas humanas de su degradación, para así trascender la explicación exclusivamente basada en el cambio climático. Este estudio es el primero en abordar la conexión entre las tasas del transporte de sedimentos en el río Magdalena y los niveles de deforestación en la cuenca mediante la aplicación de un modelo numérico (BQART), cuya eficiencia se ha demostrado a nivel global (Syvitski \& Milliman, 2007) y que ya ha sido calibrado para el Magdalena, con el fin de obtener los valores de erosión debidos a la deforestación en diferentes escalas de tiempo.

\section{Controles naturales y antrópicos en la erosión de cuencas globales}

Durante la última década, los estudios a nivel mundial sobre la evaluación, la cuantificación y la modelación numérica de la producción de sedimentos han resaltado la importancia de combinar factores naturales y antrópicos al evaluar la magnitud de los procesos de erosión y transporte de sedimentos (Verstraeten \& Poesen, 2001; Walling \& Fang, 2003; Verstraeten, et al., 2003). Estos ejercicios deben combinar y relacionar parámetros topográficos, climáticos e hidrológicos (Hovius, 1998), con parámetros antrópicos estimados a partir de los cambios en los usos del suelo o en el reconocimiento de campo (Verstraeten, et al., 2003).

En Colombia se ha adelantado una iniciativa piloto del International Geosphere Biosphere Programme - Land Ocean Interaction in the Coastal Zone (IGBP-LOICZ) en el contexto suramericano (Restrepo \& Kjerfve, 2002; Kjerfve, et al., 2002), y en el marco del proyecto del departamento del Magdalena, la universidad Eafit, Colciencias y Cormagdalena denominado "Río Magdalena - Descargas de agua y sedimentos al Mar Caribe: causas naturales, variabilidad interanual, tendencias y consideraciones sobre sus implicaciones ambientales en las zonas costeras”, con la cual se exploraron inicialmente los factores naturales que controlan la descarga de sedimentos al mar Caribe. Aun cuando los resultados del proyecto son de gran importancia para el país en el contexto de la formulación de los planes de ordenamiento territorial (POT), de los de manejo ambiental y los de navegabilidad, todavía no se han evaluado el efecto y la magnitud que los factores antrópicos tienen sobre la erosión. En el contexto mundial, las evaluaciones de los factores que controlan la producción de sedimentos (erosión) se han aplicado ampliamente en los siguientes ámbitos: estudios de navegabilidad en sistemas fluviales; determinación de la vida útil de los embalses; delimitación de zonas fuente de producción de sedimentos y diseño de planes de control de la erosión; planes regionales para el control de la deforestación y el manejo de áreas naturales protegidas, y manejo de ecosistemas vulnerables (en este sentido, cabe citar los estudios "Ecosistemas de los Andes colombianos" del Instituto Alexander von Humboldt, y el "Programa de Desarrollo Sostenible Ecoandino" del Ministerio del Medio Ambiente, Vivienda y Desarrollo Territorial).

A escala global, los estudios sobre producción de sedimentos (erosión) se han orientado a identificar los parámetros físicos que controlan dicho proceso. Algunos autores han señalado que la producción de sedimentos es controlada, en gran medida, por la actividad tectónica y la configuración del relieve, en tanto que los parámetros ambientales (es decir, la precipitación, la escorrentía y la temperatura), juegan un papel secundario al explicar la variación en la producción de sedimentos (Ahnert, 1970; Ahnert, 1984; Stallard, 1988; Pinet \& Souriau, 1988; Milliman \& Syvitski, 1992). Partiendo de este enfoque, Milliman \& Syvitski (1992) señalan que las mayores tasas de erosión $\left(>5.000 \mathrm{t} \mathrm{km}^{-2}\right.$ $\mathrm{a}^{-1}$ ) se presentan en pequeñas cuencas del sudeste Asiático y Oceanía caracterizadas por relieves altos y escarpados y por intensa actividad tectónica. Otros autores afirman que 
la producción de sedimentos depende de la precipitación media anual, con una respuesta ampliamente regulada por la cobertura vegetal y el tipo de clima dominante (Langbein \& Schumm, 1958; Fournier, 1960; Douglas 1967; Holeman, 1968; Wilson, 1973; Jansen \& Painter, 1974; Jansson, 1982; Ohmori, 1983).

Otras investigaciones (Summerfield \& Hulton, 1994; Ludwig \& Probst, 1998; Hovius, 1998; Harrison, 2000), sugieren que la producción de sedimentos es más una función de un conjunto combinado de parámetros físicos que de un solo factor en particular. En un contexto global, Ludwig \& Probst (1998) encontraron que la producción de sedimentos es controlada por la escorrentía, la variación estacional de la precipitación y la pendiente del terreno, mientras que Hovius (1998) indicó que el conjunto de variables más eficientes para explicar la varianza en la producción de sedimentos está conformado por el área de la cuenca, la altura máxima, la escorrentía, la temperatura promedio y la variación estacional de la temperatura.

Actualmente, los niveles de erosión y transporte fluvial rara vez representan cantidades o procesos naturales en cuencas hidrográficas. Durante los últimos cien años los ríos han estado sometidos a un impacto ambiental permanente. En los países en desarrollo, en donde están presentes la mayoría de los grandes ríos, ha habido un incremento considerable en los tensores de origen antrópico, incluida la minería, la deforestación, los proyectos de ingeniería, la agricultura y los cambios de usos del suelo, entre otros (Vörösmarty \& Meybeck, 2000; Meybeck, 2002).

La conversión de bosques en pastos para la ganadería y en zonas agrícolas altera el ciclo hidrológico y modifica las propiedades físicas y químicas del suelo (es decir, el contenido de materia orgánica, la porosidad, la tasa de infiltración), lo que da como resultado una mayor susceptibilidad frente a los procesos de meteorización y erosión (Bruijnzeel, 1990). Walling \& Fang (2003) reportan en Asia incrementos hasta del $80 \%$ en el transporte de sedimentos en un periodo de 30 años como resultado de la deforestación y la expansión de la frontera agrícola, y señalan que tales variaciones pueden ser mayores ( 2 a 5 veces) dependiendo de la litología y las condiciones climáticas del área intervenida. También se ha indicado que los efectos de la conversión de los bosques sobre el transporte de sedimentos se reflejan con mayor eficacia en pequeñas cuencas, ya que la proporción de áreas intervenidas con respecto al área de drenaje es alta y existe una menor probabilidad de que los sedimentos sean almacenados dentro de la cuenca (Walling \& Fang, 2003; Syvitski, 2003). Por ejemplo, para las cuencas tributarias del río Yangtze (China), que tienen altas tasas de erosión $\left(\sim 3500 \mathrm{t} \mathrm{km}^{-2} \mathrm{a}^{-1}\right)$, Higgitt \& Lu (2001a) indican que el $60 \%$ de la descarga de sedimentos es atribuible a la deforestación y la expansión de cultivos en zonas de ladera, mientras que Yang, et al. (2002) señalan que estos tensores antrópicos causaron un incremento en la producción de sedimentos del $12 \%$ en la década de 1970 y un cambio en el ciclo anual de transporte.

Los primeros intentos por relacionar tensores antrópicos y tasas de erosión se deben a Douglas (1967), quien con base en el estudio de 22 cuencas australianas indicó que frente a una cantidad igual de escorrentía, las diferencias en los niveles de erosión ( $\left.\mathrm{km}^{-2} \mathrm{a}^{-1}\right)$ eran el resultado del grado de perturbación del suelo derivado de actividades agrícolas y ganaderas. En estudios regionales (Dunne, 1979; Krishnaswamy, et al., 2001) se demuestra que existe una relación directa entre el aporte de sedimentos al sistema fluvial y el uso del suelo, la cual se hace más marcada en zonas agrícolas y ganaderas con el aumento de la escorrentía. Con base en el análisis de cuencas tributarias del río Yangtsé (China), Higgitt \& Lu (2001b) señalan que la erosión en esta cuenca se explica mediante parámetros físicos, poblacionales y variables de los usos del suelo. Mediante el estudio y la manipulación de series hidrológicas (escorrentía-transporte de sedimentos), Walling \& Fang (2003), por su parte, evaluaron el efecto del componente antrópico sobre la producción de sedimentos $\left(\mathrm{t} \mathrm{km}^{-2} \mathrm{a}^{-1}\right)$ en 145 cuencas alrededor del mundo, sugiriendo que en los países en desarrollo se presentan tendencias ascendentes en los niveles de erosión. Combinando parámetros físicos y variables de usos del suelo, Verstraeten, et al. (2003) obtuvieron un modelo multivariado que explicaba el $30 \%$ de la erosión en España, y, posteriormente, incorporando al modelo un índice factorial de ponderación (factor del Scoring Index) basado en el reconocimiento de campo de variables como la litología, la cobertura vegetal y las clases de cultivos, lograron desarrollar modelos numéricos que explicaban el $78 \%$ de la erosión $\left(\mathrm{t} \mathrm{km} \mathrm{k}^{-2} \mathrm{a}^{-1}\right)$.

\section{Factores naturales en el control de la erosión del río Magdalena}

Desde hace diez años, un grupo de investigadores del Departamento de Geología de la Universidad Eafit, con el apoyo de Colciencias, Cormagdalena y la Universidad de Colorado en Estados Unidos, han venido analizando las causas y las tendencias (1980-2000) de la erosión en el río Magdalena (Restrepo, 2005; Restrepo \& Syvitski, 2006; Restrepo, et al., 2006a, 2006b; Restrepo, 2008; Restrepo, et al., 2009; Kettner, et al., 2010). Una de las principales preguntas que se plantearon fue por qué la tasa de erosión del río Magdalena (690 ton $\mathrm{km}^{-2}$ año-1, valor estimado en la estación de Calamar en el 2000) era la más alta del continente en comparación con los grandes ríos suramericanos como el Amazonas (167 ton $\mathrm{km}^{-2}$ año-1), el Orinoco (158 ton $\mathrm{km}^{-2}$ año${ }^{1}$ ), el Paraná (43 ton $\mathrm{km}^{-2}$ año-1 y el Sao Francisco (10 ton $\mathrm{km}^{-2}$ año-1). En teoría, varios factores naturales explicarían estos altos valores en el Magdalena: el relieve, reflejado en cuencas montañosas con altas pendientes; la gran actividad tectónica; las diferencias espaciales en la composición de 
los suelos; un clima con grandes variaciones de temperatura y rangos de precipitación entre los 500 y $6.000 \mathrm{~mm}$ al año, así como la capacidad de transporte de sedimentos de los ríos por sus caudales entre moderados y altos (Restrepo \& Kjerfve, 2000a; 2000b).

El análisis espacial de la erosión en 32 sistemas tributarios principales y más de 50 estaciones de aforo en la cuenca del Magdalena (Figura 1), con series de datos sobre transporte de sedimentos entre los 10 y los 26 años, indica que el promedio de erosión en toda la cuenca es de 690 ton $\mathrm{km}^{-2}$ año ${ }^{-1}$, con valores máximos hasta de 2.200 ton $\mathrm{km}^{-2}$ año${ }^{1}$ en la cuenca del río Carare, una de las tasas de erosión más altas a nivel global (Restrepo, et al., 2006a). Los sitios críticos o hot spots corresponden a sistemas de la cuenca oriental como la del Carare, la del Opón (1.975 ton $\mathrm{km}^{-2}$ año${ }^{1}$ ) y la del Lebrija (1.260 ton $\mathrm{km}^{-2}$ año-), y a ríos como el Negro (1.730 ton $\mathrm{km}^{-2}$ año-1 $)$, La Miel (1.250 ton $\mathrm{km}^{-2}$ año-1 ), el Saldaña (1.270 ton $\mathrm{km}^{-2}$ año-1 $)$, el Coello (1.035 ton $\mathrm{km}^{-2}$ año ${ }^{-1}$ ) y el Cauca (830 ton $\mathrm{km}^{-2}$ año-1) (Restrepo, et al., 2006a). Este análisis de la distribución espacial de la erosión no evaluó las causas y los factores humanos en la degradación de los suelos de cada cuenca (Tabla 1) como producto del cambio en su uso, incluidos aspectos como la deforestación, la ganadería, la minería, la urbanización y la construcción de infraestructura.

El análisis de correlación estadística entre 30 variables hidrológicas, climáticas y morfométricas calculadas para 32 sistemas tributarios del río Magdalena, incluido el río Cauca, y la producción de sedimentos, indicó que el 58 \% de la erosión se debía a la escorrentía $(\Delta f)$ y al caudal máximo $\left(\mathrm{Q}_{\max }\right)$ (Restrepo \& Syvitski, 2006). En general, la erosión natural se debe en gran parte a los caudales máximos aportados durante eventos de corta duración. Esto podría indicar que los suelos de la cuenca tienen menos capacidad para "amortiguar" o regular las precipitaciones. La relación entre el caudal y las precipitaciones interanuales, tanto el promedio de lluvia como los eventos extremos de precipitación, no han sido analizados para la cuenca como tampoco para las zonas críticas con alta degradación en los suelos. Suponiendo que la hipótesis de que las actividades humanas en la cuenca del Magdalena (principalmente la deforestación), son la causa principal en el incremento de procesos hidrogeológicos de alto riesgo como las inundaciones y los deslizamientos, sería de esperar que las relaciones lineales entre la precipitación y el caudal, y entre el caudal y el transporte de sedimentos, se apartarían de las tendencias teóricas iniciales. Es decir, que con la alteración en los suelos, una precipitación dada (que no se incrementara en el tiempo) produciría más caudal y transporte de sedimentos que los que se generaban antes de la intervención.

\section{Simulación de las causas naturales y humanas en la erosión del río Magdalena (1980-2000)}

El modelo BQART es un modelo analítico con algoritmos empíricos diseñado para predecir las tasas de transporte de sedimentos en ríos debidas a la interacción de factores como la geomorfología, la geología, la geografía y la influencia humana (Syvitski \& Milliman, 2007):

$\overline{Q s}=\omega B \bar{Q}^{0.31} \mathrm{~A}^{0.5} R \bar{T}$ para cuencas con $\bar{T} \geq 2^{\circ} \mathrm{C}$, donde $B$ se define como: $B=I L\left(1-T_{E}\right) E_{H}, \overline{Q s}$ es el promedio interanual de sedimentos en suspensión $\left(\mathrm{kg} \mathrm{s}^{-1}\right), \bar{Q}$ es el promedio interanual del caudal $\left(\mathrm{km}^{3} \mathrm{a}^{-1}\right)$, $A$ es el área de la cuenca $\left(\mathrm{km}^{2}\right), R$ es el relieve máximo $(\mathrm{km}), \bar{T}$ es la temperatura promedio espacial de la cuenca $\left({ }^{\circ} \mathrm{C}\right)$, $\omega$ es una constante de proporcionalidad $(0,02), L$ es el factor litológico de la cuenca (-), $T_{E}$ es la eficiencia de captura de sedimentos en embalses, ciénagas y planos de inundación (-) y $E_{h}$ es la

Tabla 1. Factores naturales que controlan la erosión en la cuenca del río Magdalena (Restrepo \& Syvitski, 2006; Restrepo, et al., 2006a)

\begin{tabular}{|c|c|c|c|}
\hline Clasificación/Modelo-Ecuación & $\mathbf{r}^{2}$ & Valor $\mathbf{p}$ & Prueba $\mathbf{F}$ \\
\hline \multicolumn{4}{|l|}{ Cuenca } \\
\hline (1) $\log S y=-0,8838+0,8140 \log R-0,3906 \log Q_{\max }$ & 0,5840 & $0,0001^{\mathrm{c}}$ & 16,15 \\
\hline \multicolumn{4}{|l|}{ Cuenca alta } \\
\hline (2) $S y=107,092+0,4227 Q_{\max }$ & 0,7513 & $0,0001^{\mathrm{c}}$ & 33,23 \\
\hline \multicolumn{4}{|l|}{ Cuenca media } \\
\hline (3) $\mathrm{Sy}=3484,95-0,5042 \mathrm{H}-38,1722 \mathrm{Hr}-2,3837 \mathrm{Q}$ & 0,7704 & $0,0241^{\mathrm{b}}$ & 6,71 \\
\hline \multicolumn{4}{|l|}{ Cordillera Central } \\
\hline (4) $\log \mathrm{Sy}=1,3576+0,4401 \log \mathrm{A}$ & 0,4823 & $0,0122^{\mathrm{b}}$ & 9,32 \\
\hline \multicolumn{4}{|l|}{ Cordillera Occidental } \\
\hline (5) $\log \mathrm{Sy}=12,733-2,1389 \log \mathrm{H}+0,7755 \log \mathrm{Q}-0,9718 \log \mathrm{P}-0.4048 \log \mathrm{A}$ & 0,8950 & $0,0001^{c}$ & 202,13 \\
\hline
\end{tabular}

$\mathrm{r}^{2}=$ coeficiente de determinación de la regresión múltiple; $\mathrm{p}=$ valor $\mathrm{p}$ de probabilidad sobre la variable independiente; ${ }^{\mathrm{a}}=$ significativo con $90 \%$ de confianza;

$\mathrm{b}=$ significativo con $95 \%$ de confianza; ${ }^{\mathrm{c}}=$ significativo con $99 \%$ de confianza.

Sy = producción de sedimentos $\left(\mathrm{t} \mathrm{km}^{-2} \mathrm{a}^{-1}\right) ; \mathrm{R}=$ escorrentía; $\mathrm{Q}=$ caudal promedio interanual; $\mathrm{Q}_{\max }=$ promedio de caudales máximos interanuales; $\mathrm{A}=$ área; $\mathrm{P}=$ precipitación promedio interanual; $\mathrm{H}=$ altura promedio de la cuenca; $\mathrm{Hr}=$ coeficiente entre la altura máxima y la longitud de la cuenca 
influencia humana, ajustada numéricamente a los índices de deforestación para la cuenca del Magdalena (Kettner, et al., 2010).

Este modelo BQART ya se había implementado para la cuenca del río Magdalena en el marco de un proyecto conjunto de la Universidad Eafit y la Universidad de Colorado en Estados Unidos financiado con fondos de la NASA para la modelación de la erosión continental (Kettner, et al., 2010). La variación espacial y temporal de los datos climatológicos, hidrológicos, geológicos, litológicos y de deforestación se obtuvieron de las bases de datos de estudios anteriores de la Universidad Eafit (Restrepo, 2005; Restrepo \& Syvitski, 2006; Restrepo, et al., 2006a; Restrepo, 2008) y de nuevos datos del Instituto de Hidrología, Meteorología y Estudios
Ambientales de Colombia (Ideam) sobre tasas de transporte de sedimentos y caudales con corte al año 2000.

En general, el modelo BQART sobreestimó el transporte de sedimentos en 21 sistemas tributarios del Magdalena en un 25 \% (Tabla 2). Un 50 \% de la erosión en la cuenca del Magdalena es de origen natural, y se explica por variables hidrológicas, del relieve y climáticas. El término $B$, que en litología se refiere a la eficiencia de la retención, y en la variable humana a indicadores de deforestación, explicó un $32 \%$ adicional del transporte de sedimentos para toda la cuenca. El $82 \%$ de los datos simulados se ajustó bien a los datos observados (Figura 2). Para cualquiera de las cuencas del Magdalena, el modelo simuló bien el transporte de sedimentos dentro de un factor de desviación de 3.

Tabla 2. Factores naturales y de origen humano empleados para simular la variabilidad espacial del transporte de sedimentos en la cuenca del río Magdalena (Kettner, et al., 2010)

\begin{tabular}{|c|c|c|c|c|c|c|}
\hline Tributario & $\begin{array}{c}\text { Caudal } \\
\left(\mathrm{km}^{3} \mathrm{a}^{-1}\right)\end{array}$ & $\begin{array}{l}\text { Área } \\
\left(\mathbf{k m}^{2}\right)\end{array}$ & $\begin{array}{c}\text { Relieve } \\
\text { (km) }\end{array}$ & $\begin{array}{l}\text { Temp } \\
\left({ }^{\circ} \mathrm{C}\right)\end{array}$ & $\begin{array}{l}\text { Transporte de sedimentos } \\
\text { observado }{ }^{\text {a }}\left(\mathrm{Mt} \mathrm{a}^{-1}\right)\end{array}$ & $\begin{array}{l}\text { Transporte de sedimentos } \\
\text { simulado }{ }^{\mathrm{b}}\left(\mathrm{Mt} \mathrm{a}^{-1}\right)\end{array}$ \\
\hline Río Magdalena & 226,3 & 257.438 & 5,4 & 21,8 & $144,2 \pm 48,37$ & $183-207$ \\
\hline 2. Suaza & 1,4 & 1.014 & 1,6 & 17,9 & $0,57 \pm 0,35$ & 0,18 \\
\hline 3. Páez & 5,7 & 4.760 & 3,6 & 12,9 & $2,89 \pm 1,44$ & 1,17 \\
\hline 7. Cabrera & 2,2 & 2.713 & 3,6 & 20,0 & $1,85 \pm 1,00$ & 4,20 \\
\hline 9. Sumapaz & 1,3 & 2.433 & 3,7 & 10,8 & $0,50 \pm 0,38$ & 0,84 \\
\hline 10. Bogotá & 1,2 & 5.409 & 2,9 & 12,3 & $1,32 \pm 1,28$ & 5,29 \\
\hline 11. Coello & 1,3 & 1.041 & 3,5 & 17,1 & $1,64 \pm 2,34$ & 1,06 \\
\hline 13. Recio & 0,6 & 643 & 4,7 & 4,3 & $0,16 \pm 0,11$ & 0,05 \\
\hline 16. Gualí & 0,7 & 458 & 4,6 & 12,7 & $0,19 \pm 0,10$ & 0,15 \\
\hline 17. Guarino & 1,0 & 840 & 2,9 & 14,9 & $0,45 \pm 0,46$ & 0,24 \\
\hline 18. La Miel & 7,7 & 2.363 & 2,5 & 19,9 & $2,66 \pm 1,43$ & 3,28 \\
\hline 19. Negro & 4,3 & 4.575 & 3,3 & 21,9 & $7,97 \pm 6,75$ & 6,11 \\
\hline 20. Cocorná & 1,8 & 790 & 2,1 & 27,6 & $0,59 \pm 0,64$ & 0,47 \\
\hline 22. Samaná & 5,7 & 1.713 & 2,7 & 18,7 & $0,93 \pm 0,35$ & 0,52 \\
\hline 23. Nare & 12,5 & 5.564 & 2,9 & 21,3 & $2,59 \pm 1,53$ & 0,95 \\
\hline 24. Carare (bajo) & 8,3 & 4.909 & 3,6 & 20,1 & $16,76 \pm 8,85$ & 17,45 \\
\hline 24a. Carare (alto) & 7,3 & 1.607 & 3,1 & 19,1 & $10,87 \pm 5,09$ & 3,87 \\
\hline 25. Opón & 2,8 & 1.752 & 1,9 & 22,8 & $3,35 \pm 1,70$ & 7,68 \\
\hline 27. Suárez(bajo) & 9,5 & 9.775 & 3,7 & 13,9 & $3,41 \pm 1,93$ & 8,80 \\
\hline 27a. Suárez(alto) & 6,2 & 5.115 & 2,9 & 11,7 & $2,64 \pm 1,06$ & 3,80 \\
\hline 28. Fonce & 2,7 & 2.083 & 3,2 & 14,0 & $0,57 \pm 0,22$ & 1,80 \\
\hline 29. Sogamoso & 13,7 & 21.211 & 3,7 & 12,3 & $11,22 \pm 5,75$ & 33,70 \\
\hline 30. Cauca & 75,2 & 66.751 & 4,2 & 21,6 & $49,05 \pm 11,7$ & 49,73 \\
\hline 31. Cesar & 1,7 & 18.827 & 1,8 & 25,0 & $0,15 \pm 0,07$ & 0,10 \\
\hline Sumatoria subcuencas & & & & & 121 & 151,43 \\
\hline
\end{tabular}

Los números de los tributarios indican la codificación de las subcuencas para el análisis espacial.

${ }^{a}$ Valores observados de transporte anual de sedimentos y desviación estándar

${ }^{\mathrm{b}}$ Valores simulados después de aplicar el modelo BQART incluyendo el término $B$ 
En conclusión, el modelo BQART fue calibrado para el Magdalena con un excelente ajuste estadístico y puede usarse para simulaciones y proyecciones de la erosión por cambio climático o influencias humanas directas en la cuenca (Kettner, et al., 2010).

Con base en la estimación de la variable antrópica expresada en los índices de deforestación para el periodo 1980-2000 (Kettner, et al., 2010), el área de la cuenca del Magdalena con valores críticos de erosión representa el 78 \% (Figura 3). En síntesis, tres cuartas partes de la cuenca andina más grande del país están en estado de erosión (incluso algunos investigadores lo llaman estado de "desertificación").
Estos índices de erosión se calcularon con una ponderación numérica que incluyó el área de deforestación entre 1980 y 2000 para cada sistema tributario, asignando valores numéricos diferentes de acuerdo con el porcentaje de variación de la superficie de bosques en el periodo de 20 años (Kettner, et al., 2010). Sin embargo, estos valores de erosión no han sido calculados con las tasas de deforestación actualizadas, por ejemplo, las disponibles después de la última evaluación de deforestación para el periodo 20002010 llevada a cabo por el Ideam en el año 2010. Estos indicadores numéricos de erosión tampoco se han simulado para condiciones previas a la intervención humana en la cuenca y para futuros escenarios con diferentes estados de intervención antrópica.

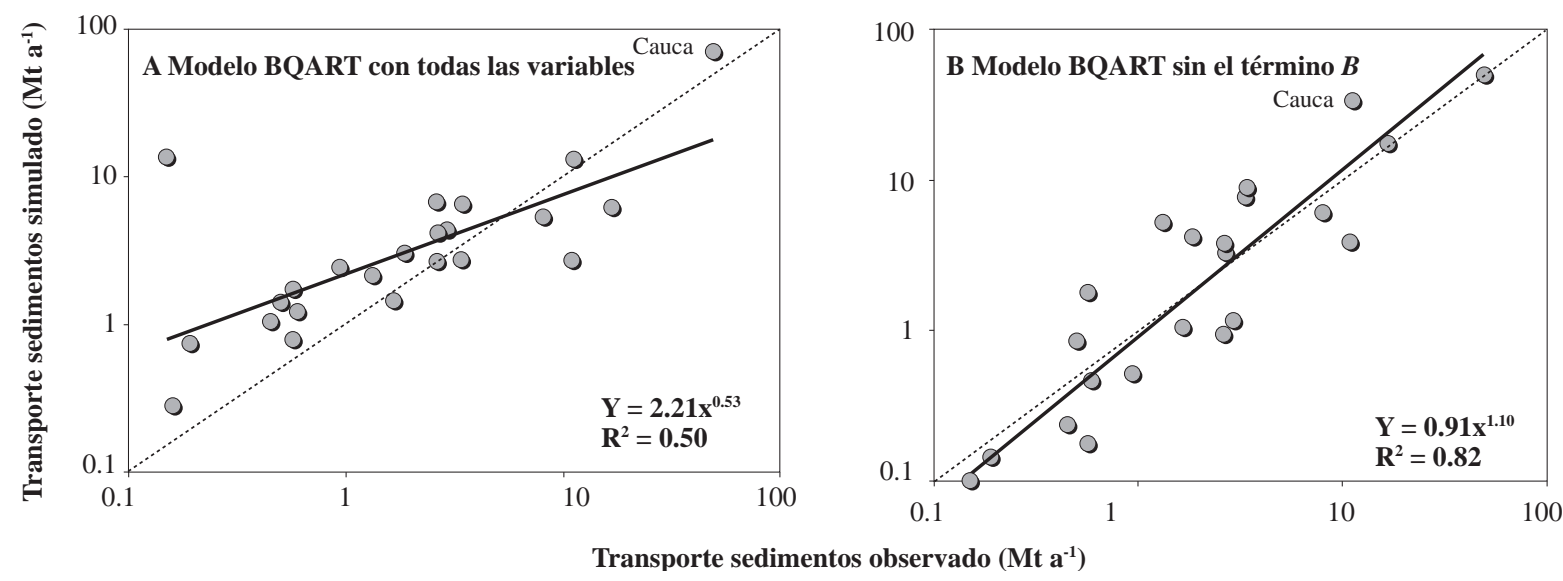

Figura 2. Datos observados de transporte de sedimentos en 23 cuencas tributarias del río Magdalena para el periodo 1980-2000 comparados con los datos simulados del modelo BQART sin incluir litología, eficiencia de retención de sedimentos y deforestación (A) y valores observados comparados con los simulados después de incluir el término $B$ con las anteriores variables (B) (modificado de Kettner, et al., 2010).

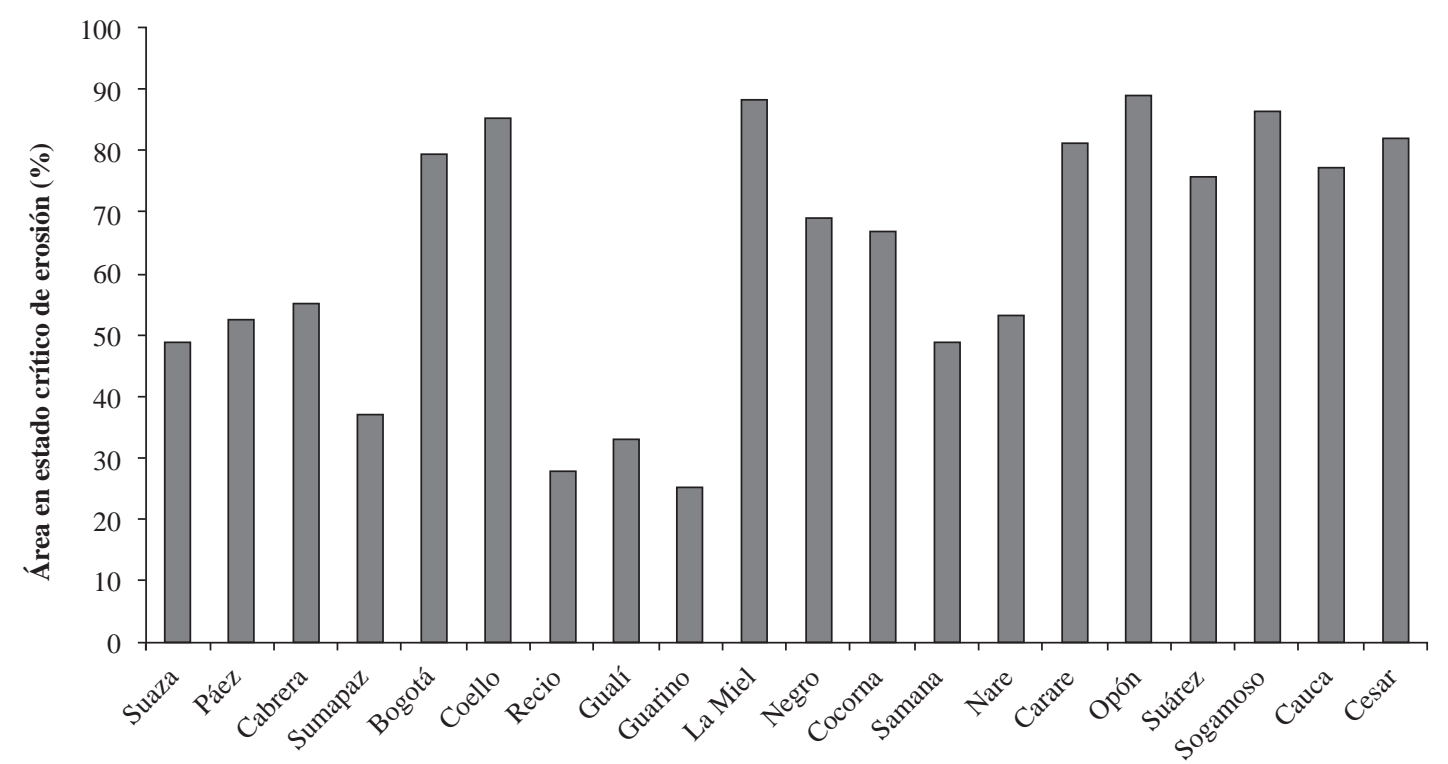

Figura 3. Porcentaje de área en estado de erosión para algunos sistemas tributarios del río Magdalena (datos de Kettner, et al., 2010). 


\section{Simulación de la erosión debida a la deforestación (1980-2010)}

Con base en las metodologías de análisis utilizadas en el estudio de erosión en el río Magdalena (Restrepo, 2005; Restrepo, et al., 2006a, 2006b; Restrepo \& Syvitski, 2006, Restrepo, 2008; y Kettner, et al., 2010), se siguieron los siguientes pasos para la simulación del transporte de sedimentos durante el periodo 1980-2010: 1) el cálculo de las variables hidrológicas, climáticas, morfométricas y litológicas para cada subcuenca, actualizando los valores de caudal y transporte de sedimentos con corte al año 2010; 2) estimación de las áreas de deforestación en los periodos de 1980-2000 y 2000-2010 en cada subcuenca tributaria del Magdalena (Tabla 2), con base en los estudios de deforestación en la cuenca para el periodo 1980-2000 de Restrepo (2005), Restrepo \& Syvitski (2006) y del Ideam para el de 2000-2010 a escala nacional. Los análisis de las bases de datos de deforestación, tanto las del programa ArcInfo del estudio de transporte de sedimentos de la Universidad Eafit (Restrepo, 2005; Restrepo \& Syvitski, 2006; Restrepo, et al., 2006a), como las del estudio del Ideam para el periodo 2000-2010 se analizaron en el laboratorio SIG de la Universidad Eafit (Ideam, 2011); 3) implementación del modelo numérico BQART (caudal, área de cuenca, temperatura, máxima elevación, litología, depósito y deforestación), ya calibrado para la cuenca del Magdalena, y 4) estimación del ajuste estadístico del modelo BQART en cada subcuenca tributaria (valores medidos versus valores simulados) y cálculo del porcentaje de varianza explicado por el modelo en relación con la deforestación en los periodos 1980-2000 y 2000-2010.

El análisis del cambio en la cobertura forestal de la cuenca del Magdalena entre 1980 y 2000 indica que aproximadamente el $43 \%$ del área de bosques fue talada (Figura 4). Gran parte de estos suelos fueron transformados en áreas de agricultura y ganadería, de hecho, las áreas de bosque transformadas en este periodo se duplicaron. La tasa de deforestación anual fue de 2,1\%, valor de deforestación reportado como uno de los más altos de cuencas tropicales a nivel mundial (Restrepo \& Syvitski, 2006) (Figura 4 A y B).

Otras evaluaciones sobre la deforestación en el Magdalena han mostrado que hasta el 2000 las áreas naturales de bosques eran del orden de 23 \% (evaluación de deforestación de The Nature Conservancy - TNC, 2012) y de al menos $10 \%$ en el año 2005, valor este basado en el mapa de ecosistemas de la cuenca del Magdalena levantado por el Instituto Humboldt (Restrepo, 2005) (Figura 1S, http://www.raccefyn.co/index.php/raccefyn/ article/downloadSuppFile/141/745). En general, desde la década de los años setenta, las tasas de deforestación se han incrementado casi exponencialmente sin mostrar ninguna desaceleración en las tres últimas décadas.

Los valores de deforestación para los periodos 1980-2000 y 2000-2010 se acoplaron al modelo BQART para la simulación del transporte de sedimentos en el periodo 1980-

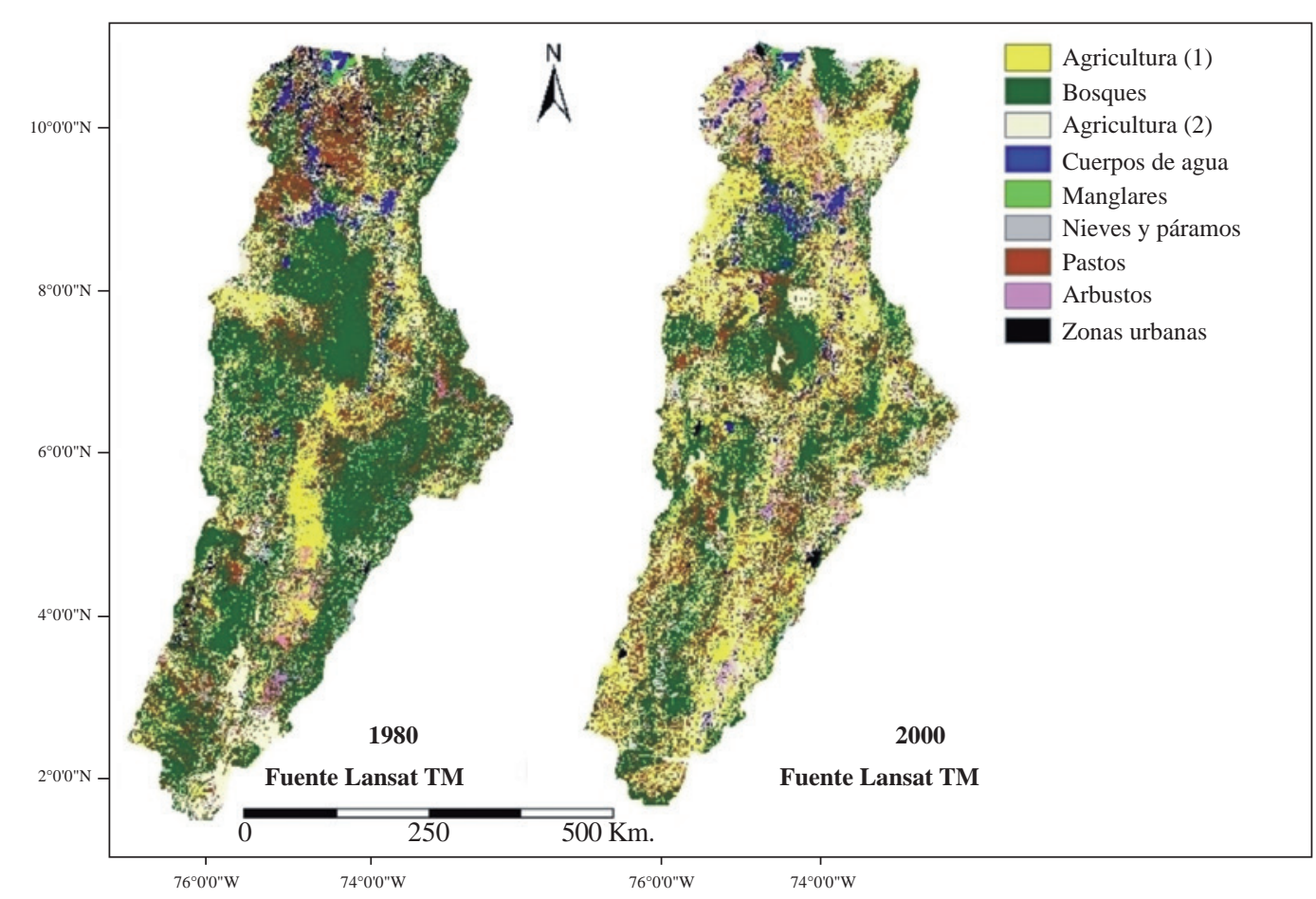

Figura 4. Mapas de cambios en el uso del suelo en el cuenca del río Magdalena para el periodo 1980-2000 derivados de la clasificación de imágenes Landsat (modificado de Restrepo \& Syvitski, 2006). 
2010 (Tabla 3). Los resultados para la cuenca del Magdalena mostraron que el modelo explicó el 86 \% de la varianza en el transporte de sedimentos una vez todas las variables naturales y humanas se incluyeron en la simulación; la varianza correspondiente a los aportes de sedimentos sin incluir $E_{h}$, y la influencia antrópica ajustada numéricamente con los índices de deforestación fue de 77 \%. En otras palabras, la deforestación explicó un $9 \%$ del transporte de sedimentos en la cuenca durante las últimas tres décadas (Figura 5).

El análisis entre décadas de la sumatoria de los aportes simulados de sedimento debidos a la deforestación en cada subcuenca tributaria indicó que durante las tres últimas décadas un total de $417 \mathrm{Mt}$ de sedimentos fueron generadas por las actividades de tala de bosques (Tabla 4). Para la última década (2000-2010), la producción combinada de sedimentos de la cuenca por deforestación fue de 160 Mt, valor cercano al transporte anual del Magdalena en la estación más aguas abajo de Calamar.

La producción anual de sedimentos en el Magdalena por deforestación durante la década de 2000 al 2010, de $16 \mathrm{Mt} \mathrm{a}^{-1}$, es cercana al aporte anual del río Patía, que es el valor de descarga de sedimentos más alto de todo el Pacífico americano (Restrepo \& Kettner, 2012; Restrepo, 2012). En otras palabras, debido a la deforestación, el río Magdalena transporta cada año lo correspondiente a un río Patía a las zonas bajas de inundación de la Depresión

Tabla 3. Factores naturales y humanos usados para simular la variabilidad espacial del transporte de sedimentos en la cuenca del río Magdalena para el periodo 1980-2010

\begin{tabular}{|c|c|c|c|c|c|c|c|}
\hline Tributario & $\begin{array}{c}\text { Caudal } \\
\left(\mathbf{k m}^{3} \mathrm{a}^{-1}\right) \\
1980-2010\end{array}$ & $\begin{array}{l}\text { Área } \\
\left(\mathbf{k m}^{2}\right)\end{array}$ & $\begin{array}{c}\text { Relieve } \\
\text { (km) }\end{array}$ & $\begin{array}{c}\text { Temp } \\
\left({ }^{\circ} \mathrm{C}\right)\end{array}$ & $\begin{array}{c}\text { Transporte de } \\
\text { sedimentos } \\
\text { observado }{ }^{\mathrm{a}}\left(\mathrm{Mt} \mathrm{a}^{-1}\right) \\
1980-2010\end{array}$ & $\begin{array}{c}\text { Transporte de } \\
\text { sedimentos } \\
\text { simulado }^{\mathrm{b}}\left(\mathrm{Mt} \mathrm{a}^{-1}\right) \\
1980-2010\end{array}$ & $\begin{array}{c}\text { Transporte de } \\
\text { sedimentos simulado } \\
\left(\mathrm{Mt} \mathrm{a}^{-1}\right) E_{h}=1 \\
1980-2010\end{array}$ \\
\hline Río Magdalena & 245 & 257.438 & 5,4 & 21,8 & 188,2 & 149 & \\
\hline 2. Suaza & 1,4 & 1.014 & 1,6 & 17,9 & 0,47 & 0,18 & 0,59 \\
\hline 3. Páez & 5,5 & 4.760 & 3,6 & 12,9 & 2,98 & 2,71 & 3,87 \\
\hline 7. Cabrera & 2,2 & 2.713 & 3,6 & 20,0 & 1,55 & 4,2 & 6 \\
\hline 9. Sumapaz & 1,4 & 2.433 & 3,7 & 10,8 & 0,38 & 0,86 & 2,85 \\
\hline 10. Bogotá & 1,3 & 5.409 & 2,9 & 12,3 & 1,35 & 5,43 & 3,88 \\
\hline 11. Coello & 1,2 & 1.041 & 3,5 & 17,1 & 1,16 & 1,05 & 0,75 \\
\hline 13. Recio & 0,7 & 643 & 4,7 & 4,3 & 0,16 & 0,05 & 0,16 \\
\hline 16. Gualí & 0,8 & 458 & 4,6 & 12,7 & 0,26 & 0,15 & 0,5 \\
\hline 17. Guarino & 1,6 & 840 & 2,9 & 14,9 & 0,57 & 0,28 & 0,93 \\
\hline 18. La Miel & 7,8 & 2.363 & 2,5 & 19,9 & 2,32 & 3,29 & 2,35 \\
\hline 19. Negro & 4,4 & 4.575 & 3,3 & 21,9 & 7,09 & 6,17 & 8,82 \\
\hline 20. Cocorná & 1,8 & 790 & 2,1 & 27,6 & 0,49 & 0,47 & 0,66 \\
\hline 22. Samaná & 5,8 & 1.713 & 2,7 & 18,7 & 0,9 . & 0,53 & 1,76 \\
\hline 23. Nare & 12,8 & 5.564 & 2,9 & 21,3 & 2,25 & 0,96 & 1,37 \\
\hline 24. Carare & 8,4 & 4.909 & 3,6 & 20,1 & 15,04 & 17,52 & 12,51 \\
\hline 25. Opón & 2,8 & 1.752 & 1,9 & 22,8 & 3,29 & 7,74 & 5,53 \\
\hline 27. Suárez & 9,4 & 9.775 & 3,7 & 13,9 & 3,3 . & 17,58 & 12,56 \\
\hline 28. Fonce & 2,7 & 2.083 & 3,2 & 14,0 & 0,63 & 1,8 & 2,58 \\
\hline 29. Sogamoso & 13,8 & 21.211 & 3,7 & 12,3 & 10,91 & 33,73 & 24,09 \\
\hline 30. Cauca & 76,0 & 66.751 & 4,2 & 21,6 & 48,51 & 50,1 & 17,79 \\
\hline 31. Cesar & 1,7 & 18.827 & 1,8 & 25,0 & 0,14 & 0,1 & 0,1 \\
\hline Sumatoria subcuencas & & & & & 104 & 155 & 110 \\
\hline
\end{tabular}

Los números de los tributarios indican la codificación de las subcuencas para el análisis espacial.

${ }^{\text {a }}$ Valores observados de transporte anual de sedimentos para el periodo 1980-2010

b Valores simulados después de aplicar el modelo BQART incluyendo el término B para el periodo 1980-2010

C Valores simulados sin incluir la variable humana por deforestación en el término B $\left(E_{h}=1\right)$ para el periodo 1980-2010 


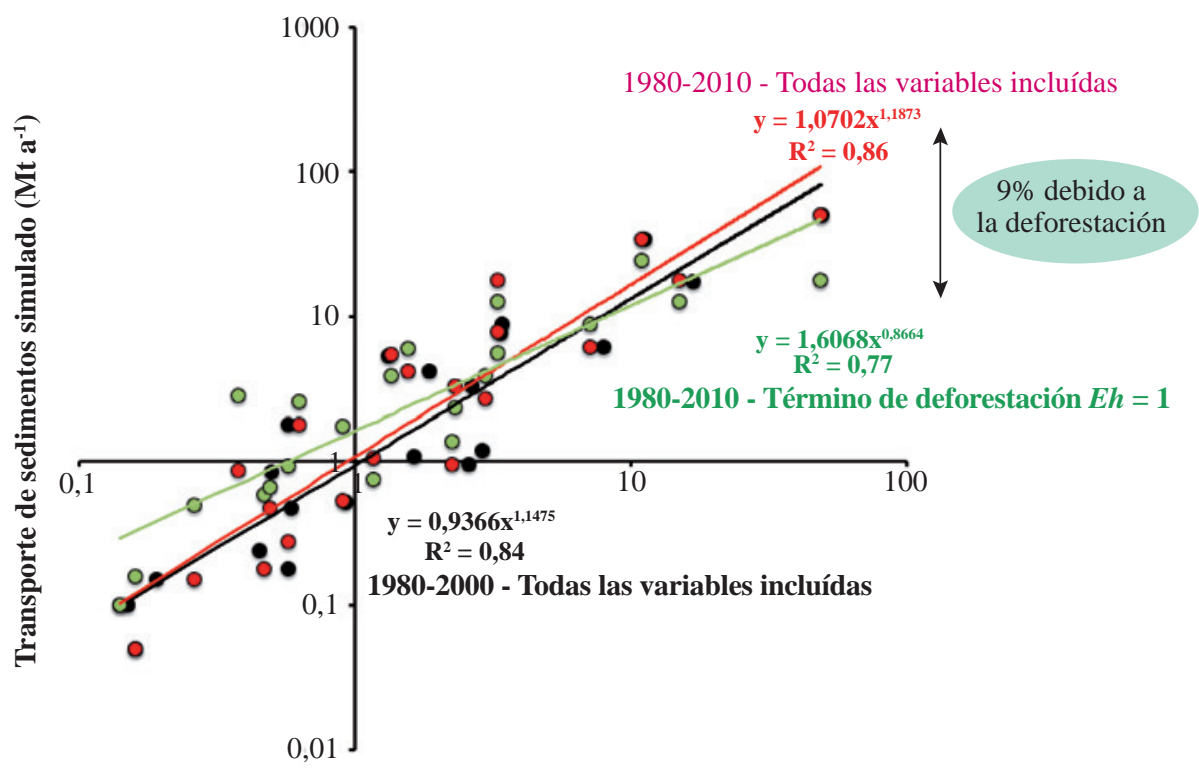

Transporte de sedimentos observado (Mt $\left.\mathrm{a}^{-1}\right)$

Figura 5. Datos observados de transporte de sedimentos en 23 cuencas tributarias del río Magdalena comparados con los datos simulados del modelo BQART para los periodos 1980-2000 (color negro) y 1980-2010 (color rojo), después de incluir las variables naturales y humanas. La simulación sin incluir el término de deforestación $\left(E_{h}=1\right)$ para el intervalo 1980-2010 se indica con color verde.

Momposina, el Canal del Dique y su delta en Bocas de Ceniza. También es importante resaltar que las cuencas tributarias como la del Cauca, el Suárez, el Sogamoso y el Carare han transportado durante los últimos diez años un orden de magnitud más de sedimentos en comparación con los otros tributarios (Tabla 4).

\section{Tendencias del transporte de sedimentos del río Magdalena, 1970-2011}

Los siguientes análisis de la serie de transporte de sedimentos del Magdalena en la estación más aguas abajo de Calamar para el periodo 1972-2011, se presentan como una herramienta para establecer conexiones temporales entre décadas de las posibles relaciones entre tendencias e impactos humanos en la cuenca.

Para identificar y evaluar la significancia estadística de las tendencias interanuales en el caudal y el transporte de sedimentos del Magdalena en Calamar, se hicieron pruebas de Mann-Kendall (TMK) en las series. Esta es una prueba no-paramétrica que no exige un tipo o función de distribución específico en la serie de datos (Yue, et al., 2002). La prueba se ha usado ampliamente para detectar tendencias lineales de series hidrológicas, especialmente de caudal, y pude emplearse como un indicador del cambio hidrológico en sistemas fluviales (Yue, et al., 2002; Restrepo, et al., 2014, entre otros). Los resultados estadísticos de las pruebas en las series del Magdalena se muestran en la tabla 5.

Según los resultados de estas pruebas, las tendencias ascendentes en los aportes fluviales fueron todas estadísticamente representativas. Los incrementos en el caudal en el periodo entre 1940 y 2011, así como en el transporte de sedimentos entre 1972 y 2011 fueron significativos para toda la extensión de las series (Tabla 5, Figura 2S, http://www.raccefyn.co/index.php/raccefyn/article/ downloadSuppFile/141/746). Para los intervalos de tiempo posteriores al año 2000, tanto las pendientes Sen, que expresan la magnitud del cambio por unidad de tiempo, como los promedios interanuales de caudales y transporte de sedimentos, se incrementaron considerablemente en las descargas del Magdalena y del Dique en comparación con los valores anteriores al año 2000 (Tabla 5). De hecho, las tendencias de los aportes fueron mucho más pronunciadas para el periodo 2005-2011. Por ejemplo, el caudal promedio de $7.156 \mathrm{~m}^{3} \mathrm{~s}^{-1}$ para el periodo 1940-1999, se incrementó a $8.833 \mathrm{~m}^{3} \mathrm{~s}^{-1}$ entre los años 2005 y 2011, un aumento de 1.677 $\mathrm{m}^{3} \mathrm{~s}^{-1}$ o del $24 \%$ con respecto al promedio interanual de las serie completa del periodo 1940-2011. En relación con el transporte de sedimentos, estas tendencias ascendentes fueron igualmente representativas, mostrando un aumento de 100.000 ton día ${ }^{-1}$ para el periodo 2005-2011 en relación con el promedio del periodo 1972-1999 (Tabla 5).

Entre el 2005 y el 2011, el transporte anual del Magdalena en Calamar se incrementó en un 32 \% con respecto al promedio del periodo 1970-2000, es decir, un aumento de 44 millones de toneladas por año (Figura 6). Este ascenso en el transporte coincide con las tendencias ascendentes de deforestación en Colombia entre el 2005 y el 2010 (ver sección siguiente). Al convertir el transporte de sedimentos anual del Magdalena en Calamar en número de volquetas de seis toneladas y dado un valor específico de densidad 
Tabla 4. Total de transporte de sedimentos simulado durante los periodos 1980-2000 y 1980-2010 en cada subcuenca tributaria del río Magdalena. Se muestran las cantidades totales durante la última década (2000-2010) y los valores debidos a la deforestación.

\begin{tabular}{|c|c|c|c|}
\hline Tributario & $\begin{array}{c}\text { Transporte total de } \\
\left.\text { sedimentos (Mt } 20 \mathrm{a}^{-1}\right) \\
1980-2000\end{array}$ & $\begin{array}{c}\text { Transporte total de } \\
\left.\text { sedimentos (Mt } 30 \mathrm{a}^{-1}\right) \\
1980-2010\end{array}$ & 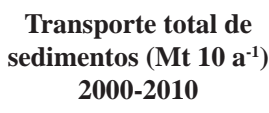 \\
\hline 2. Suaza & 3,6 & 5,4 & 1,8 \\
\hline 3. Páez & 23,4 & 81,3 & 57,9 \\
\hline 7. Cabrera & 84 & 126 & 42 \\
\hline 9. Sumapaz & 16,8 & 25,8 & 9 \\
\hline 10. Bogotá & 105,8 & 162,9 & 57,1 \\
\hline 11. Coello & 21,2 & 31,5 & 10,3 \\
\hline 13. Recio & 1 & 1,5 & 0,5 \\
\hline 16. Gualí & 3 & 4,5 & 1,5 \\
\hline 17. Guarino & 4,8 & 8,4 & 3,6 \\
\hline 18. La Miel & 65,6 & 98,7 & 33,1 \\
\hline 19. Negro & 122,2 & 185,1 & 62,9 \\
\hline 20. Cocorna & 9,4 & 14,1 & 4,7 \\
\hline 22. Samana & 10,4 & 15,9 & 5,5 \\
\hline 23. Nare & 19 & 28,8 & 9,8 \\
\hline 24. Carare & 349 & 525,6 & 176,6 \\
\hline 25. Opón & 153,6 & 232,2 & 78,6 \\
\hline 27. Suárez & 176 & 527,4 & 351,4 \\
\hline 28. Fonce & 36 & 54 & 18 \\
\hline 29. Sogamoso & 674 & 1011,9 & 337,9 \\
\hline 30. Cauca & 994,6 & 1503 & 508,4 \\
\hline 31. Cesar & 2 & 3 & 1 \\
\hline Sumatoria subcuencas & 2875,4 & 4647,2 & 1771,6 \\
\hline Deforestación $^{\mathrm{a}}$ & 230,0 & 418,2 & 159,5 \\
\hline
\end{tabular}

${ }^{a}$ Transporte total de sedimentos debido a la deforestación después de calcular el 9\% de la varianza explicada por el factor Eh en el término B del modelo BQART.

Tabla 5. Resultados del test de Mann Kendall (TMK) para los promedios mensuales de las series de caudal y transporte de sedimentos del río Magdalena en Calamar antes y después del año 2000

\begin{tabular}{|c|c|c|c|c|c|c|c|c|c|c|}
\hline \multirow{2}{*}{$\begin{array}{l}\text { Estación- } \\
\text { Variable }\end{array}$} & \multicolumn{5}{|c|}{ Antes del 2000} & \multicolumn{5}{|c|}{ Después del 2000} \\
\hline & $\begin{array}{l}\text { Primer } \\
\text { año }\end{array}$ & $\begin{array}{l}\text { Último } \\
\text { año }\end{array}$ & Tau-K & Pendiente Sen & Promedio & $\begin{array}{l}\text { Primer } \\
\text { año }\end{array}$ & $\begin{array}{c}\text { Último } \\
\text { año }\end{array}$ & Tau-K & Pendiente Sen & Promedio \\
\hline Calamar Q & 1940 & 1999 & 0,082 & $13.538 \mathrm{~m}^{3} / \mathrm{s} / \mathrm{a}$ & 7.156 & 2000 & 2011 & 0,535 & $527.643 \mathrm{~m}^{3} / \mathrm{s} / \mathrm{a}$ & 7,.783 \\
\hline Calamar Qs & 1972 & 1999 & 0,163 & 4.830 t/día/a & 394.346 & 2000 & 2011 & 0,373 & 30.185 t/día/a & 413.898 \\
\hline Calamar Q & .. & $\ldots$ & ... & $\ldots$ & ... & 2005 & 2011 & 0,222 & $599.500 \mathrm{~m}^{3} / \mathrm{s} / \mathrm{a}$ & 8.833 \\
\hline Calamar Qs & $\ldots$ & $\ldots$ & $\ldots$ & $\ldots$ & $\ldots$ & 2005 & 2011 & 0,221 & 47.793 t/día/a & 495.794 \\
\hline
\end{tabular}

$\mathrm{Q}=$ caudal en $\mathrm{m}^{3} \mathrm{~s}^{-1} ; \mathrm{Q}_{\mathrm{s}}=$ transporte de sedimentos en ton día $\mathrm{a}^{-1} ; \mathrm{t}=$ toneladas de sedimentos en suspensión; $\mathrm{s}=$ segundos; $\mathrm{a}=$ año

de los sedimentos, el Magdalena transportó en Calamar 15 millones de volquetas anuales o 40.000 volquetas por día, para un promedio de 1.650 volquetas por hora. Por otra parte, el análisis de las desviaciones del transporte de sedimentos del Magdalena en Calamar para el periodo 1970-2011, indica que aproximadamente el $60 \%$ de las desviaciones positivas se presentó entre el 2000 y el 2011. Estos resultados preliminares indican que el Magdalena 


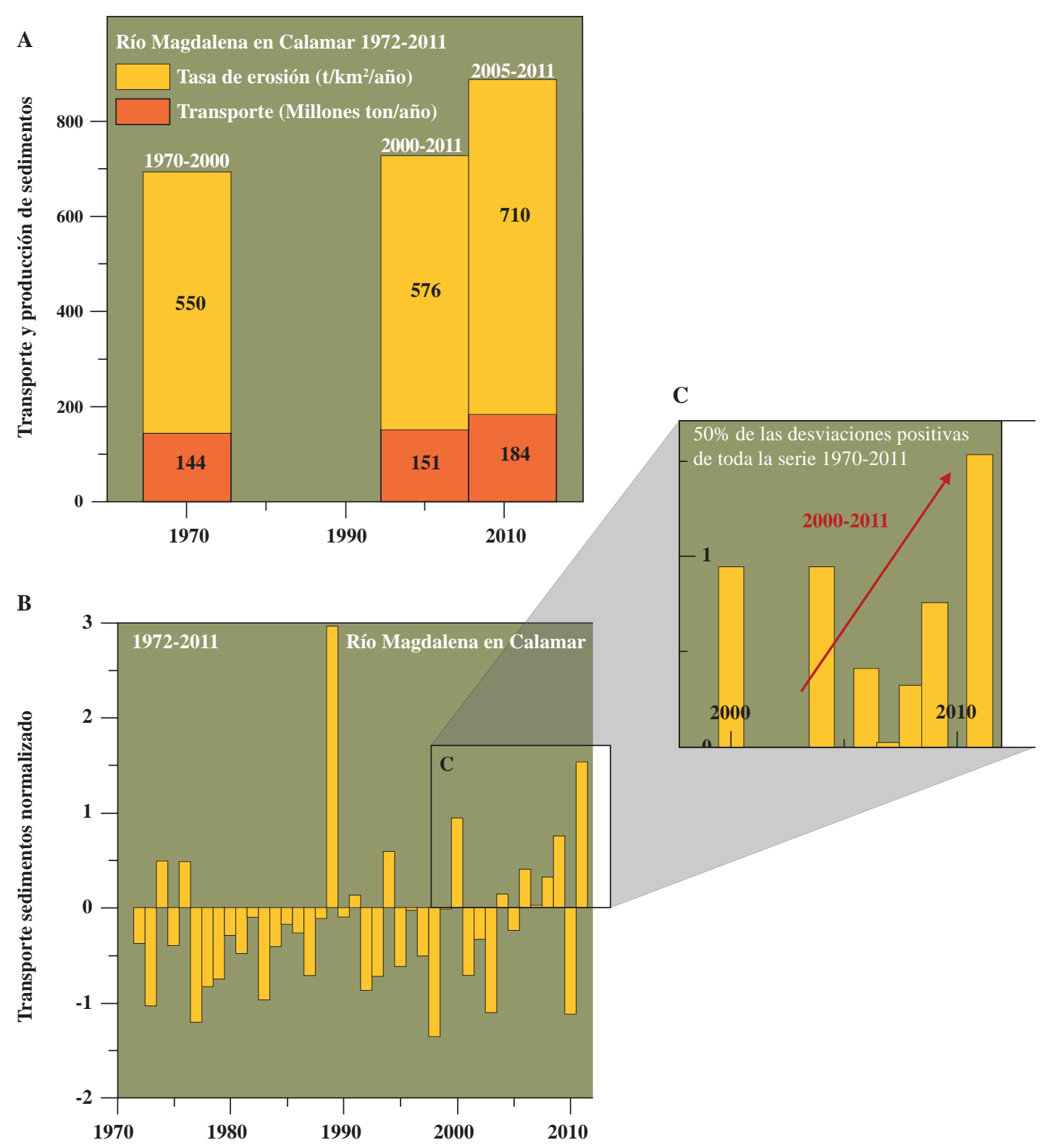

Figura 6. Aportes de sedimentos en suspensión y producción de sedimentos del río Magdalena en la estación Calamar para las décadas 1972-2000 y 2000-2011 (A) y serie de tiempo de transporte en suspensión normalizado entre 1972 y 2011 (B-C).

es el sistema fluvial del continente americano con las tasas de erosión más altas por kilómetro cuadrado de área hidrográfica para el periodo 2005-2011.

\section{Indicadores ambientales y económicos en el río Magdalena}

El desempeño ambiental de Colombia en la última década se ha medido y registrado en varias bases de datos globales en términos de índices de desempeño, cambios forestales y conflictos ambientales. El índice de desempeño ambiental (Environmental Performance Index- EPI) de la Universidad de Yale clasifica a Colombia en el puesto 85 a nivel global, con una calificación general de 50,77 sobre 100 (EPI, 2014). En las áreas de manejo y cambio en las coberturas de los bosques, como también de los recursos hídricos, las calificaciones de 26,26 y 4,6, respectivamente, dan muestra de la degradación ambiental de las cuencas hidrográficas. Por su parte, la base global de deforestación, Global Forest Watch (GFW, 2014), indica que solo queda el $14 \%$ de la cobertura forestal primaria en todo el país. El área total de deforestación en el 2012 fue de 197.000 ha. Por último, el Atlas Global de Justicia Ambiental (EJA, 2014) sitúa a Colombia en el puesto número dos de países con mayores conflictos ambientales. Los datos muestran que en el país han ocurrido 72 conflictos ambientales generados principalmente por las actividades extractivas de recursos mineros, biomasa forestal y energía fósil. 
Con relación a las actividades de deforestación, el estudio global de cuencas fluviales del Instituto Mundial de los Recursos (WRI) (Revenga, et al., 2000), señala que la cobertura de bosques en la cuenca del Magdalena era del $90 \%$ antes de los asentamientos humanos. Hoy en día, los bosques no superan el $10 \%$ en cobertura de acuerdo con la evaluación de ecosistemas en la cuenca del Magdalena, trabajo realizado por el Instituto Humboldt y publicado en el libro Los Sedimentos del Magdalena: Reflejo de la Crisis Ambiental (Restrepo, 2005).

Los indicadores de deforestación en Colombia son alarmantes. La última evaluación de deforestación hecha por el Ideam entre los años 2000 y 2010 muestra una tasa anual de deforestación de 336.000 hectáreas por año, un área de pérdida forestal similar al área del departamento del Atlántico. Al comparar este valor con los datos de deforestación global publicados en el estudio de evaluación mundial de los bosques de la Organización para la Alimentación y la Agricultura de las Naciones Unidas (FAO, 2010), Colombia, que ocupa solo el 0,1 \% del área continental del mundo, representa el 5 \% de la deforestación global. En otras palabras, nuestro país está en los primeros 10 lugares de deforestación a nivel mundial. No cabe duda de que eso contribuye a la degradación de nuestros suelos, influye en la erosión e incrementa el transporte de sedimentos de los ríos colombianos, incluido el de su máximo exponente, el río Magdalena (Restrepo, 2013).
Al observar los indicadores económicos en la cuenca del Magdalena que generan cambio en el uso de los suelos, incluida la agricultura, la minería, la urbanización y la electricidad, es evidente que en términos de su contribución al PIB nacional, la agricultura, seguida por la urbanización, son las actividades de origen humano que han transformado en mayor escala los suelos de la cuenca del Magdalena. Estas transformaciones comenzaron en la década de los setenta en el sector agrícola y en los años ochenta con la extracción minera (Figura 7).

Diversas evaluaciones sobre la deforestación en países tropicales han resaltado cómo gran parte de la pérdida forestal en los trópicos se debe a la transformación de los suelos en zonas agrícolas. Geist \& Lambin (2002) reportan que el $96 \%$ de la tala de bosques en Latinoamérica se debe a las actividades agrícolas. En un reciente análisis de más de 117 estudios econométricos, Ferretti-Gallón \& Busch (2014) encontraron que el 58 \% de la deforestación global actual en los trópicos ocurre debido al mayor retorno económico de la agricultura y el pastoreo.

De acuerdo con el Atlas de Justicia Ambiental (EJA, 2014), existe una clara conexión entre el número e intensidad de los conflictos ambientales y el modelo económico extractivo de los últimos gobiernos en Colombia. La inversión foránea pasó de 1.444 millones de dólares en 1994 a 15.612 millones en el 2012. Por ejemplo, el sector
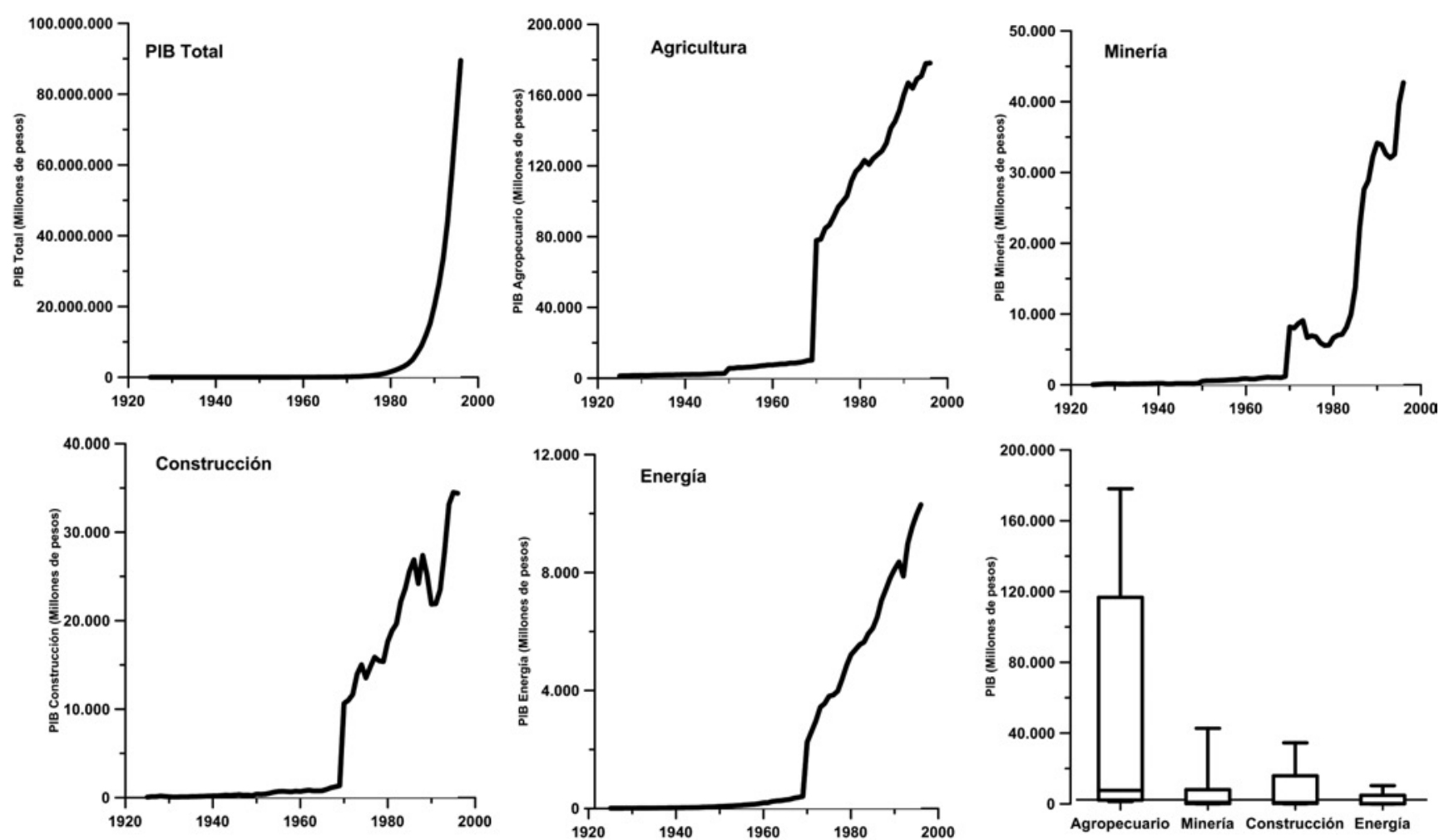

Figura 7. Contribución al PIB nacional de las actividades de origen humano que generan cambios en el uso de los suelos en la cuenca del río Magdalena para el periodo 1935-2000 (datos de indicadores económicos del Banco de la República). 
extractivo de recursos naturales evidenció un incremento de 14 a $55 \%$ en el mismo periodo. El sector minero aumentó su participación en el PIB nacional de 2 a $11 \%$ entre los años 1979 y 2012. En general, el $64 \%$ del total de las exportaciones del país en la última década se generó en el sector minero-energético.

No se han hecho análisis de los costos de la degradación de los suelos y, por ende, de la erosión en Colombia. En el reporte del Banco Mundial sobre las prioridades ambientales para la reducción de la pobreza en Colombia, SánchezTriana, et al. (2007) señalan que los costos de la erosión de los suelos podrían estar en el orden de los 2 billones por año. Sin embargo, los autores afirman que estos valores serían incluso mucho mayores debido a la escasez de bases de datos sobre los deslizamientos y las inundaciones y de otros cálculos asociados con los servicios ecosistémicos y de infraestructura. Por su parte, la ex Viceministra de Medio Ambiente y Desarrollo Sostenible, Adriana Soto (revista Semana Sostenible, junio de 2014), estimaba que los costos del deterioro de una hectárea de suelo son del orden de 5.000 dólares. Debido a que entre 1999 y 2010 se han deforestado cerca de 6 millones de hectáreas, el valor total de su restauración sería de cerca de 30 billones de dólares, aproximadamente el 8 \% del PIB nacional del 2013.

Dados los indicadores económicos y de población, no sería desacertado afirmar que gran parte de estas realidades ambientales son el resultado principalmente de las actividades humanas en el Magdalena. De acuerdo con The Nature Conservancy, $86 \%$ del PIB proviene de las actividades en la cuenca, representadas a escala nacional por el $75 \%$ de la producción agrícola, el 70 \% de la hidroelectricidad, el $90 \%$ de la energía térmica y el 80 \% de la producción de carbón. De hecho, 30 millones de habitantes están en la cuenca, es decir, cerca del 80 \% de la población del país.

La figura 3S, http://www.raccefyn.co/index.php/raccefyn/ article/downloadSuppFile/141/747, resume las tendencias del transporte de sedimentos del río Magdalena para el periodo 1972-2011 en la estación de aforo más aguas abajo, Calamar, localizada antes de la confluencia con el Canal del Dique y con el delta en Bocas de Ceniza. Los datos de esta estación resumen el comportamiento general de los pulsos y tendencias de los aportes fluviales provenientes de las partes media y alta de la cuenca. Aunque no dan una medida de la variabilidad espacial aguas arriba, sí representan el comportamiento acumulado de todos los procesos de producción y de depósito de sedimentos.

En general, las tendencias ascendentes más representativas se dieron a mediados de los años setenta, a finales de los ochenta y noventa, y especialmente en la última década, del 2002 al 2011. Todos estos periodos coinciden con los incrementos en los indicadores económicos (Figura 7) y con el aumento en las tasas de deforestación en Colombia para el periodo 2005-2010 (Figura 1S).
Las implicaciones de la erosión del río Magdalena en las inundaciones de la Depresión Momposina: ¿cambio climático o actividades humanas?

La planicie aluvial de la Depresión Momposina, sobre la cual confluyen las descargas fluviales de los ríos y tributarios del Cauca, Magdalena y San Jorge, es una de las grandes zonas inundables del mundo. Se trata de una especie de "delta interior", con una extensión de 2.850 $\mathrm{km}^{2}$. Esta depresión tectónica, compuesta por sistemas de ciénagas, quebradas, caños y humedales, desempeña un papel regulador o de "amortiguador hídrico" de toda la región del bajo Magdalena. Estas llanuras de inundación se expanden al absorber el exceso de agua en el sistema durante las temporadas de invierno o lluvias intensas y se contraen a medida que liberan agua lentamente durante el verano o la estación más seca, con el fin de mantener el flujo de la corriente. La gran destrucción de los humedales y los planos de inundación del río Magdalena, producto de actividades humanas como la alteración hidráulica, el desvío de cauces para irrigación de zonas de agricultura, así como el aumento de las tasas de sedimentación causado por la minería del oro en el bajo Cauca, la construcción de obras civiles y los procesos erosivos en la parte media y alta de la cuenca del Magdalena, ha dado como resultado el deterioro de un mecanismo natural de control y regulación de los flujos extremos y las inundaciones. Gran parte de los eventos extremos de inundación en regiones como Mompox, Magangué, Calamar y Barranquilla están sujetos a la acción de esta "esponja fluvial".

El problema de las inundaciones y su recurrencia en la Depresión Momposina se debe a la gran cantidad de sedimentos que se almacenan en esta región, producto de la erosión en el río Magdalena. Una cifra preliminar del estudio de la Universidad Eafit y la Universidad de Colorado indica que entre el 20 y $45 \%$ de los sedimentos del sistema de los ríos Magdalena-Cauca-Cesar quedan retenidos en la Depresión cada año, una cifra que fluctúa entre 36 y 80 millones de toneladas anualmente. No cabe duda de que el impacto ambiental de la erosión disminuye la capacidad de carga hidrológica de las lagunas de la Depresión y La Mojana, y por lo tanto, genera eventos de inundación más pronunciados y recurrentes.

Las lluvias que se presentaron durante la ola invernal del periodo 2010-2011 fueron el resultado de la anomalía climática del fenómeno de la Niña y la localización de la banda nubosa de la zona de convergencia intertropical (Hoyos, et al., 2013). Todavía existe gran controversia científica sobre si los cambios en la frecuencia y la magnitud de los eventos del Niño o la Niña en las últimas cinco décadas son resultado del cambio climático. De lo que no cabe duda alguna es de la capacidad de regulación hídrica que tienen los suelos y los bosques. En zonas andinas con relieve muy pronunciado, los bosques y sus suelos son la esponja hídrica que almacena el exceso de precipitación 
y amortigua de forma natural la escorrentía que fluye en el ciclo hidrológico hacia los ríos. En estos sistemas montañosos de altas pendientes, la remoción de la cobertura forestal deja expuestos los suelos a la acción de las lluvias y al lavado activo o erosión superficial. En otras palabras, al remover la vegetación, los excesos hídricos en las cuencas fluviales no son filtrados y la escorrentía o caudal se presenta de forma errática o en pulsos, ocasionando las inundaciones de carácter extremo aguas abajo y el incremento en los sedimentos transportados desde las zonas activas de erosión.

Los análisis de las tendencias de los aportes fluviales en la estación Calamar demuestran que los incrementos en el caudal y el transporte de sedimentos del Magdalena han sido más pronunciados durante la última década (Tabla 5, Figuras 2S, 6 y 3S). Estos resultados coinciden con la evaluación general de los aportes de los ríos del Caribe colombiano, los cuales han presentado aumentos significativos en el caudal durante el periodo posterior al año 2000 (Restrepo, et al., 2014). Por lo tanto, los ríos que drenan los Andes del norte están experimentando ascensos en los caudales sin que sus cuencas muestren aumentos interanuales en los valores de precipitación. El análisis de las tendencias de precipitación en Colombia (Carmona \& Poveda, 2014) indica que en la región central de los Andes no existe una tendencia uniforme de aumento o reducción de la precipitación. Así, la hipótesis de que los ríos están transportando mayores niveles de escorrentía debido al cambio climático está aún por comprobarse.

Para entender si las tendencias interanuales en el caudal y el transporte de sedimentos tienen relación con el cambio climático, se deben analizar las series de tiempo de la precipitación total y de la frecuencia e intensidad de los episodios extremos de precipitación durante los últimos 50 años en cada subcuenca tributaria del Magdalena. Para relacionar espacial y temporalmente las tasas de cambio en el caudal y el transporte de sedimentos desde la cuenca del Magdalena con los indicadores de variabilidad climática como la precipitación, la temperatura del aire y los caudales, se deberían analizar los resultados recientes de las tendencias de largo plazo de series hidroclimáticas en Colombia, incluidos los de Carmona \& Poveda (2014).

En conclusión, los problemas ambientales de gran parte del país son transferidos aguas abajo y depositados en la Depresión Momposina y en La Mojana en términos de aportes de agua, sedimentos y contaminantes. La cuenca Magdalena-Cauca requiere un sistema integrado de análisis y control ambiental que incluya componentes como la deforestación, la erosión de suelos, el transporte de sedimentos y las estrategias de control y mitigación de inundaciones. Este modelo integrado, que conciba la cuenca hidrográfica como un sistema continuo desde aguas arriba hasta su desembocadura en el Caribe, daría las pautas para entender hidráulicamente el sistema de inundación y su proyección en el tiempo, información prioritaria para los planes de ordenamiento regional y ambiental. ¿Cómo controlar las inundaciones? ¿Por qué se han vuelto tan extremas? ¿Por qué la Depresión y La Mojana se inundan con más frecuencia que antes? ¿Hasta cuándo seguirá este comportamiento? ¿ $\mathrm{Se}$ intensificarán las inundaciones en el futuro? ¿Cuánto tiempo le queda a la Depresión Momposina para llenarse de sedimentos? ¿Cuáles serían las implicaciones futuras de esta situación? Las respuestas a estas preguntas que, por supuesto, no se conocen aún, serían la base para elaborar los planes nacionales de ordenamiento ambiental y mitigación de desastres.

El cambio climático y la degradación ambiental en Colombia no dan espera. Hay que empezar ya a generar las bases científicas antes de hacer obras hidráulicas y seguir enterrando miles de millones de pesos en la Depresión y La Mojana al mismo ritmo, o mayor, que la acumulación de los sedimentos provenientes de la erosión del río Magdalena.

\section{Agradecimientos}

Los estudios sobre las causas de la erosión en la cuenca del río Magdalena han sido financiados por Colciencias (2004-2008). Se ha contado con fondos de la NASA para la simulación del transporte de sedimentos mediante el proyecto Inter-disciplinary Research in Earth Science Program (NNH06ZDA001N-IDS) (2009-2010) con la Universidad de Colorado en Boulder y el proyecto de la Unesco sobre Ríos Tropicales, IGCP 582 (2007-2013). Los resultados presentados en este artículo sobre la simulación del aporte de la deforestación en el transporte de sedimentos de la cuenca del Magdalena para el periodo 1980-2010 está financiado por el proyecto actual titulado "La erosión en el río Magdalena debida a la deforestación, 1950-2010: Tasas históricas y simulación", financiado por el Fondo para la Investigación de la Ciencia y la Tecnología del Banco de la República (No.3.276, periodo 2014-2015).

\section{Información suplementaria}

Figura 1S. Mapas de coberturas boscosas en la cuenca del Magdalena y en Colombia para los periodos (A) 2000, (B) 2005 y (C) 2012 (tomado de Restrepo 2005, TNC 2012, IDEAM 2013). También se muestran las áreas de deforestación en la cuenca durante el periodo 2005-2010 con base en el análisis de deforestación IDEAM-REED en el 2014 (D).

Figura 2S. Tendencias del transporte de sedimentos del río Magdalena en Calamar para los periodos 1972-2011 (arriba), 20002011 (centro) y 2005-2011 (abajo).

Figura 3S. Serie de tiempo de transporte de sedimentos en suspensión del río Magdalena en la estación Calamar durante el periodo 1972-2011, con los valores de transporte promedio antes y después del año 2000 y para el periodo 2005-2011. 


\section{Conflicto de intereses}

El autor declara que no tiene ningún conflicto de intereses.

\section{Bibliografía}

Ahnert, F. 1970. Functional relationships between denudation, relief and uplift in large mid-latitude drainage basins. American Journal of Science 268: 243-263.

Ahnert, F. 1984. Local relief and the height limits of mountain ranges. American Journal of Science. 284: 1035-1055.

Bonachea, J., Viola, M., Bruschi, M.A., Hurtado, L., Forte, L.M., da Silva, M., Etcheverry, R., Cavallotto, J., Marcilene, F., Dantas, O., Lázaro, V., Zuquette, MA., Bezerra, O., Remondo, J., Rivas, V., Gómez-Arozamena, J., Fernández, G., Cendrero, A. 2010. Natural and human forcing in recent geomorphic change; case studies in the Rio de la Plata basin. Science of the Total Environment. 408: 2674-2695.

Bruijnzeel, L. 1990. Hydrology of moist tropical forest and effects of conversion: a state of the knowledge. UNESCO: 224.

Carmona, A.M., Poveda, G. 2014. Detection of long-term trends in monthly hydro-climatic series of Colombia through Empirical Mode Decomposition. Climate Change. doi 10.1007/s/10584-013-1046-3.

Cendrero, A., Rivas, V., Remondo, J. 2004.Influencia humana sobre los procesos geológicos superficiales; consecuencias ambientales. En Naredo, J,M., Ed., Incidencia de la especie humana sobre la Tierra. Colección Economía y Naturaleza, Fundación César Manrique, Lanzarote.

Cendrero, A., Remondo, J., Bonachea, J., Rivas, V., Soto J. 2006. Sensitivity of landscape evolution and geomorphic processes to direct and indirect human influence. Geogr Fis Geodin Quatern. 29: 125-137.

Douglas, I. 1967. Man, vegetation and the sediment yield of rivers. Nature. 215: 925-928.

Dunne, T. 1979. Sediment yield and land use in tropical catchments. Journal of Hydrology. 42: 281-300.

EJA-Environmental Justice Atlas. 2014. http://ejatlas.org/.

EPI-Environmental Performance Index. 2014. http://epi.yale. edu/epi.

Food and Agriculture Organization of the United Nations (FAO). 2010. State of the World's forests 2009. Food and Agriculture Organization of the United Nations, Report. 117.

Ferretti-Gallon, K., Busch, J. 2014. What drives deforestation and what stops it? A meta-analysis of spatially explicit econometric studies. Center for Global Development Working Paper. 361.

Fournier, F. 1960. Climat et erosion: la relation entre l'erosion du sol par l'eau et les précipitations atmosphériques. Presse Universitaire de France. 201.

Geist, H.J., Lambin, E.F. 2002. Proximate causes and underlying driving forces of tropical deforestation. Bioscience. 52: 143-150.
GWF-Global Forest Watch. 2014. http://www.globalforestwatch. org/.

Harrison, C.G.A. 2000. What factor controls mechanical erosion rates? International Journal in Earth Sciences. $\mathbf{5 3 1 .}$

Higgitt, D., Lu, X. 2001a. Sediment delivery to the three gorges: 1. Catchment controls. Geomorphology. 41: 143-156.

Higgitt, D., Lu, X. 2001b. Sediment delivery to the three gorges: 2. Local response. Geomorphology. 41: 157-169.

Holeman, J.N. 1968. Sediment yield of major rivers of the world. Water Resources Research. 4: 737-747.

Hovius, N. 1998. Controls on sediment supply by large rivers. En: Relative Role of Eustasy, Climate, and Tectonism in Continental Rocks. SEPM (Society of Sedimentary Geology) Special Publication. 59: 3-16.

Hoyos, N., Escobar, J., Restrepo, J.C., Arango, A.M., Ortiz, J. 2013. Impact of the 2010-2011 La Niña Phenomenon in Colombia, South America: The human toll of an extreme weather event. Appl. Geogr. 39: 16-25.

Instituto de Hidrología, Meteorología, y Estudios AmbientalesIDEAM. 2011. Memoria técnica de la cuantificación de la deforestación histórica nacional -escalas gruesa y fina. Instituto de Hidrología, Meteorología, y Estudios Ambientales-IDEAM. 91.

Jansson, M.B. 1982. Land erosion by water in different climates. Uppsala, Ungi Rapport 57, Department of Physical Geography, Uppsala University. 151.

Jansen, J.M.L., Painter, R.B. 1974. Predicting sediment yield from climate and topography. Journal of Hydrology. 21: 371-380

Kettner, A., Restrepo, J.D., Syvitski, J.P.M. 2010. Simulating spatial variability of sediment fluxes in an Andean drainage basin, the Magdalena River. Journal of Geology. 118: $363-379$.

Kjerfve, B., Wiebe, W., Restrepo, J.D., Kremer, H., Salomons, W. 2002. CariBas - The Caribbean Basins Perspective. En: Kjerfve, B., Kremer, H., Salomons, W., Crossland, J.M. Eds. CariBas - Activities in River Catchments and their Impacts on Coastal Systems in the Caribbean. LOICZIGBP Report. 27: 3-28.

Krishnaswamy, J., Halpin, D., Ritcher, D. 2001. Dynamics of sediment discharge in relation to land-use and hydroclimatology in a humid tropical watershed in Costa Rica. Journal of Hydrology. 253: 91-109.

Langbein, W.B., Schumm, S.A. 1958. Yield of sediment in relation to mean annual precipitation. Transactions of the American Geophysical Union. 39: 1076-1084.

Ludwig, W., Probst, J. 1998. River sediment discharge to the oceans: Present controls and global budgets. American Journal of Science. 298: 265-295.

Meybeck, M. 2002. Water quality in river systems. En: Goudie, D., Ed., Environmental Change and Human Society. Oxford University Press. 
Milliman, J.D., Syvitski, J.P.M. 1992. Geomorphic/tectonic control of sediment transport to the ocean: the importance of small mountainous rivers. Journal of Geology. 100: 525-544.

Ohmori, H. 1983. Erosion rates and their relation to vegetation from view point of world-wide distribution. Bulletin of the Department of Geography-University of Tokyo. 15: 77-91.

Pinet, P., Souriau, M. 1988. Continental erosion and large-scale relief. Tectonics. 7: 563-582.

Restrepo, J.D., Kjerfve, B. 2000a. Water discharge and sediment load from the western slopes of the Colombian Andes with focus on Rio San Juan. Journal of Geology. 108: 17-33.

Restrepo, J.D., Kjerfve, B. 2000b. Magdalena River: Interannual variability (1975-1995) and revised water discharge and sediment load estimates. Journal of Hydrology. 235: 137-149.

Restrepo, J.D., Kjerfve, B. 2002. River Discharge, Sediment Load, and Sediment Yield Estimates for the Magdalena River and Other Caribbean Rivers of Colombia: Environmental Implications. En: Kjerfve, B., Kremer, H., Salomons, W., Crossland, J.M., Eds., CariBas - Activities in River Catchments and their Impacts on Coastal Systems in the Caribbean. LOICZ-IGBP Report. 27: 86-97.

Restrepo, J.D. 2005. Los Sedimentos del Río Magdalena: Reflejo de la Crisis Ambiental. Fondo Editorial Universidad EAFIT. 267.

Restrepo, J.C., Restrepo, J.D. 2005. Efectos Naturales y Antrópicos en la Producción de Sedimentos en la Cuenca del Río Magdalena. Revista Academia Colombiana de Ciencias Físicas, Exactas y Naturales. 29: 239-254.

Restrepo, J.D., Syvitski, J.P.M. 2006. Assessing the Effect of Natural Controls and Land Use Change on Sediment Yield in a Major Andean River: The Magdalena Drainage Basin, Colombia. Ambio: a Journal of the Human Environment. 35: 44-53.

Restrepo, J.D., Kjerfve, B., Restrepo, J.C., Hermelin, M. 2006a. Factors Controlling Sediment Yield from a Major South American Drainage Basin: The Magdalena River, Colombia. Journal of Hydrology. 316: 213-232.

Restrepo, J.D., Zapata, P., Díaz, J.M., Garzón, J., García, C. 2006b. Fluvial Fluxes into the Caribbean Sea and their Impact on Coastal Ecosystems: The Magdalena River, Colombia. Global and Planetary Change. 50: 33-49.

Restrepo, J.D. 2008. Applicability of LOICZ Catchment-Coast Continuum in a Major Caribbean Basin: The Magdalena River, Colombia. Estuarine, Coastal and Shelf Science. 77: 214-229.

Restrepo, J.D., López, S.A., Restrepo, J.C. 2009. Geomorphic factors controlling sediment yield in the Andean rivers of Colombia. Latin American Journal of Sedimentology and Basin Analysis. 16: 79-92.

Restrepo, J. D. 2012. Assessing the effect of sea-level change and human activities on a major delta on the Pacific coast of northern South America: The Patía River. Geomorphology. doi:10.1016/j.geomorph.2012.02.004.

Restrepo, J.D., Kettner, A. 2012. Human induced discharge diversion in a tropical delta and its environmental implications: the Patía River, Colombia. Journal of Hydrology. 424: 124-142.

Restrepo, J.D. 2013.The perils of human activity on South American deltas: Lessons from Colombia's experience with soil erosion. En: Deltas: Landforms, Ecosystems and Human Activities. En Proceedings of HP1, IAHS-IAPSOIASPEI Assembly, IAHS Publ. 358.

Restrepo, J., Ortíz, J.C., Pierini, J., Schrottke, K., Maza, M., Otero, L., Aguirre, J. 2014. Freshwater discharge into the Caribbean Sea from the rivers of Northwestern South America (Colombia): Magnitude, variability and recent changes. Journal of Hydrology. 509: 266-281.

Revenga, C., Brunner, J., Henninger, N., Kassem, K., Payne, R. 2000. Pilot Analysis of Global Ecosystems: Freshwater Systems. World Resource Institute, 65. http://www.wri.org/ wr2000.

Rivas, V., Cendrero, A., Hurtado, M., Cabral, M., Giménez, J., Forte, L., del Río, L., Cantú, M., Becker, A. 2006. Geomorphic consequences of urban development and mining activities; an analysis of study areas in Spain and Argentina. Geomorphology. 73: 185-206.

Sánchez-Triana, E., Ahmed, K., Awe, Y. 2007. Prioridades ambientales para la reducción de la pobreza en Colombia: un análisis ambiental del país para Colombia. Informe del Banco Mundial, Direcciones para el desarrollo, medio ambiente y desarrollo sustentable. Report No. 38610: 522.

Syvitski, J. 2003. Supply and flux of sediment along hydrological pathways: Research for the 21st century. Global and Planetary Change. 810: 1-11.

Syvitski, J.P.M., Milliman, J.D. 2007. Geology, Geography, and Humans Battle for Dominance over the Delivery of Fluvial Sediment to the Coastal Ocean. The Journal of Geology. 115: $1-19$.

Syvitski, J.P.M., Kettner, A.J. 2011. Sediment Flux and the Anthropocene. Philosophical Transactions of the Royal Society. 369: 957-975.

Stallard, R. 1988. Weathering and erosion in the humid tropics. En: Lerman, A., Meybeck, M., Eds., Physical and Chemical Weathering in Geochemical Cycles. Kluwer Academic Publishers: p. 225-246.

Summerfield, M.A., Hulton, N.J. 1994. Natural controls of fluvial denudation in major world drainage basins. Journal of Geophysical Research. 99: 13871-13884.

Yue, S., Pilon, P., Cavadias, G. 2002. Power of the MannKendall and Spearman's rho test to detecting monotonic trends in hydrological series. Journal of Hydrology. 259: 254-271.

Verstraeten, G., Poesen, J. 2001. Factors controlling sediment yield from small intensively cultivated catchments in a temperate humid climate. Geomorphology. 40: 123-144. 
Verstraeten, G., Poesen, J., de Vente, J., Koninckx, X. 2003. Sediment yield variability in Spain: A quantitative and semiqualitative analysis using reservoir sedimentation rates. Geomorphology. 50: 327-348.

Vörösmarty, C.J., Meybeck, M. 2000. Riverine transport and its alteration by human activities. IGBP Newsletter. 39: 24-29.

Walling, D.E. 1999. Linking land use, erosion and sediment yields in river basins Hydrobiologia. 410: 223-240.
Walling, D.E., Fang, D. 2003. Recent trends in suspended sediment loads of the world's rivers. Global and Planetary Change. 39: 111-126.

Wilson, L. 1973. Variations in mean annual sediment yield as a function of mean annual precipitation. American Journal of Science. 273: 335-349.

Yang, S.L., Zhao, Q.Y., Baelkin, I.M. 2002. Temporal variation in the sediment load of the Yangtze River and the influences of human activities. Journal of Hydrology. 263: 56-71. 